ARTICLE

\title{
Nanopore sequencing of brain-derived full-length circRNAs reveals circRNA-specific exon usage, intron retention and microexons
}

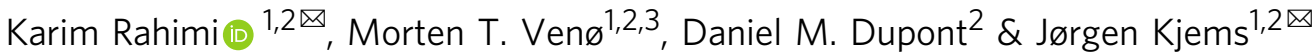

Circular RNA (circRNA) is a class of covalently joined non-coding RNAs with functional roles in a wide variety of cellular processes. Their composition shows extensive overlap with exons found in linear mRNAs making it difficult to delineate their composition using short-read RNA sequencing, particularly for long and multi-exonic circRNAs. Here, we use long-read nanopore sequencing of nicked circRNAs (circNick-LRS) and characterize a total of 18,266 and 39,623 circRNAs in human and mouse brain, respectively. We further develop an approach for targeted long-read sequencing of a panel of circRNAs (circPanel-LRS), eliminating the need for prior circRNA enrichment and find $>30$ circRNA isoforms on average per targeted locus. Our data show that circRNAs exhibit a large number of splicing events such as novel exons, intron retention and microexons that preferentially occur in circRNAs. We propose that altered exon usage in circRNAs may reflect resistance to nonsense-mediated decay in the absence of translation.

\footnotetext{
${ }^{1}$ Department of Molecular Biology and Genetics (MBG), Aarhus University, Aarhus, Denmark. ${ }^{2}$ Interdisciplinary Nanoscience Center (iNANO), Aarhus

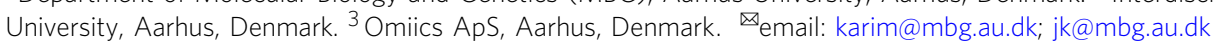


C ircular RNA (circRNA) constitutes an abundant and partially conserved group of RNAs derived from both coding and non-coding linear transcripts. They are the outcome of a unique splicing event in which the $5^{\prime}$ splice donor back-splices to the upstream $3^{\prime}$ splice acceptor, resulting in a closed circular RNA. The formation of a unique back-splice junction (BSJ) differentiates the circRNAs from their linear counterpart. CircRNAs are highly expressed in the central nervous system where many of them are regulated during development ${ }^{1-3}$, inflammation ${ }^{4}$, and also found to function as biomarkers in cancer ${ }^{5-7}$. Only a limited number of circRNAs have been characterized functionally. The most extensively studied circRNA, ciRS-7/CDR1-AS, appears to act as a regulator of microRNA miR- $7^{8-10}$ and its removal in mouse brain causes cognitive changes ${ }^{11}$.

High-throughput techniques, including Illumina-based RNAseq $^{12}$, microarrays ${ }^{13,14}$, and NanoString ${ }^{15}$ have been employed to profile circRNA expression by detecting and counting the number of unique BSJ sequences. However, none of the short-read sequencing techniques have so far been able to elucidate the exon composition of circRNAs longer than 400-500 nucleotides (nts) in the background of linear mRNA. A few studies have addressed this issue using computational analysis of paired-end reads but this has also been limited to small circRNAs ${ }^{16,17}$.

The availability of long-read sequencing techniques such as Oxford Nanopore Technology $(\mathrm{ONT})^{18}$ and Single-Molecule Real-Time ${ }^{19}$ from Pacific Biosciences (PacBio) has opened up a new era in genomic and transcriptomic studies by allowing ultralong sequencing reads ${ }^{20-23}$. ONT has been used to sequence poly (A)-purified RNA and reported a significant number of transspliced RNAs ${ }^{24}$; however, the inclusion of a poly(A) selection step prevented the detection of circRNAs. Another study combined nanopore sequencing with a PCR-based approach by using endto-end divergent primers to create BSJ reads and find different isoforms of circNPM1; however, this study was limited to this single circRNA 25 .

Here, we applied for the first time the ONT platform to provide a deep characterization of alternative splicing events in circRNAs at a global scale (see the first version of this study on bioRxiv) ${ }^{26}$. We find that mRNA and circRNA exhibit divergent exon composition with a significant number of circRNA-specific exons and splicing events seen in human and mouse brain. Strikingly, many of the so-called microexons, reported to function in development, behavior, and autism spectrum disorder, reviewed by Gonatopoulos-Pournatzis \& Blencowe ${ }^{27}$, appear to be preferentially found in circRNAs. It raises the possibility that circRNA-specific RNA elements may exert novel functions which are absent in the linear transcriptome.

\section{Results}

Nanopore long-read sequencing of global circRNA and poly(A) RNA. Only a small fraction of RNA in mammalian cells is circular $(\sim 0.1 \%$ of the rRNA-depleted RNA pool in the mouse nervous system $\left.{ }^{28}\right)$. To focus our sequencing on this small fraction of RNA, we developed a circRNA enrichment protocol with some resemblance to the RPAD protocol ${ }^{29}$ to deplete both ribosomal and linear RNA. In brief, total RNA from mouse or human brain was treated with RiboZero and RNase R to remove ribosomal and linear RNA, respectively. Since some linear RNAs are resistant to RNase $\mathrm{R}$, we conducted an additional polyadenylation step followed by poly(A)-depletion to remove the remaining linear RNA (see Fig. 1a for an overview of the full enrichment protocol). Quantification of selected circRNAs, linear mRNA, and ribosomal RNA confirmed that this protocol led to a nearly 1000 -fold enrichment of circRNAs (Supplementary Fig. 1). The circRNAenriched pool was nicked by gentle hydrolysis prior to long-read sequencing (circNick-LRS), followed by $3^{\prime}$ end polyadenylation to align with the standard ONT protocol (Fig. 1b).

Nanopore data was collected using MinKnow (v 1.0.1) software. Applying the circNick-LRS protocol to enriched circRNA from human and mouse brain samples yielded 4,273,440 and 12,683,908 high quality filtered sequencing reads, of which $189,862(4.44 \%)$ and $790,634(6.23 \%)$ contained circRNA back-splice junctions (BSJ), respectively, (Table 1). Mapping the reads to the reference genomes yielded a total of 18,266 and 39,623 unique circRNAs in human and mouse brain samples, respectively (Supplementary Data 1). Scatter plots of read length versus average read quality for ONT sequencing are shown in Supplementary Fig. 2.

To enable a global comparison to the linear mRNA transcriptome, we performed ONT full-length poly(A) RNA sequencing on the same brain samples. This resulted in a total of 21.7 and 15.4 million reads for human and mouse brain-derived RNA samples, respectively. The FLAIR tool (Full-Length Alternative Isoform analysis of RNA $)^{30}$ was used to analyze the long-read mRNA data and detect linear mRNA isoforms. In total, 42,782 isoforms derived from 9,754 genes were detected in mouse brain and 47,187 isoforms from 9,512 genes in human brain.

Long- and short-read sequencing protocols identify distinct pools of circRNAs. To investigate method-specific biases in circRNA expression profiles, we also performed conventional short-read sequencing of rRNA-depleted libraries and used a combination of the find_circ and CIRI2 pipelines for circRNA prediction $^{10,31}$. This approach produced 11,286 and 7,555 different circRNAs in human and mouse brain, respectively (detected with $>2$ reads; Supplementary Data 2 ). Comparing the circRNA profiles between long- and short-read sequencing, we found that only 3,740 and 5,299 circRNAs were detected by both methods in human and mouse brain, respectively (Fig. 1d, e). This illustrates that the benefits of long-read sequencing are not limited to elucidating internal circRNA composition but also extend to the detection of circRNAs not seen with shortread sequencing technology, at least at the sequencing depth obtained here. Our data show that highly expressed circRNAs are generally detected using both long- and short-read sequencing methods, but that each method also captures many lower expressed circRNAs not detected by the other technique (Supplementary Fig. 3).

Of the circRNAs detected by circNick-LRS, 5,537 were found in both human and mouse brain, amounting to $30.3 \%$ and $14 \%$ of the detected human and mouse circRNAs, respectively (Fig. 2a). This is on par with previous studies estimating that $10-20 \%$ of expressed circRNAs are conserved between mammalian species $^{2,32}$ and indicates that circNick-LRS is a reliable method to detect true circRNA species.

Among the highest expressed and conserved circRNAs, we found well-known brain circRNAs such as circRims1, circRims2, circHipk3, circCdyl, and circZfp609 using both methods (Fig. 2b). Surprisingly, ciRS-7, previously shown to be highly expressed in human and mouse brain, was missing in the mouse dataset, suggesting that it is unintentionally depleted in the circRNA enrichment protocol, likely due to the presence of internal poly (A) stretches in the circRNA sequence (see discussion). Figure $2 \mathrm{c}$ shows the exon map of human-specific circRNA circKHDRBS3 with a side-by-side comparison of sequencing reads generated by short-read, long-read circRNA, and long-read linear poly(A) RNA sequencing. Note that there is an exon exclusively expressed in the circRNA. Interestingly, 2,851 and 11,380 circRNAs from human and mouse, respectively, were not previously annotated in circBase $^{33}$, circAtlas ${ }^{34}$, or CIRCpedia ${ }^{35}$ (marked as novel circRNAs in Table 1 and can be found in Supplementary Data 1). 
a

circRNA enrichment

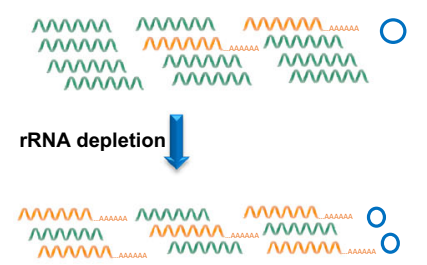

$\begin{gathered}\text { RNAse R } \\ \text { treatment }\end{gathered}$
mum

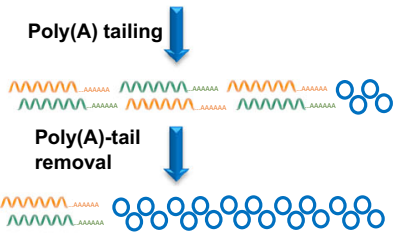

Circular RNA

Mun Non poly(A) RNA (rRNA, tRNA, etc.)

Mun Poly(A) RNA b

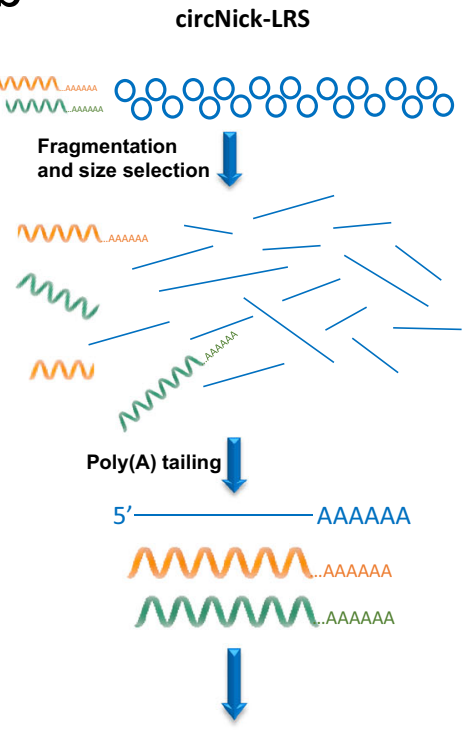

ONT library prep and long-read sequencing
C

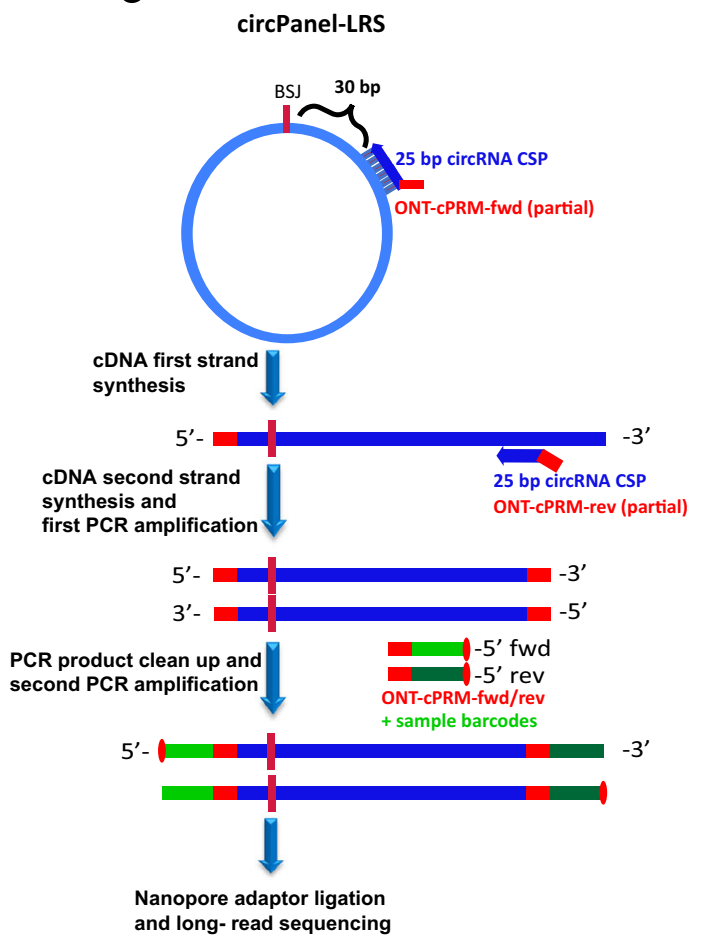

d

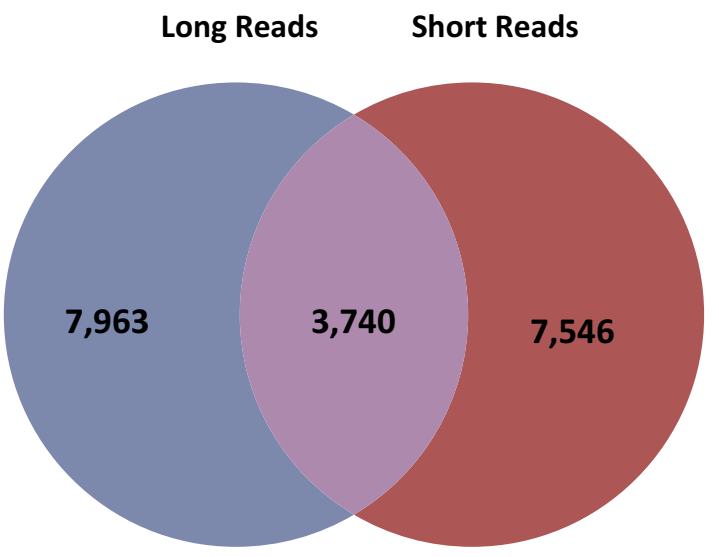

Human

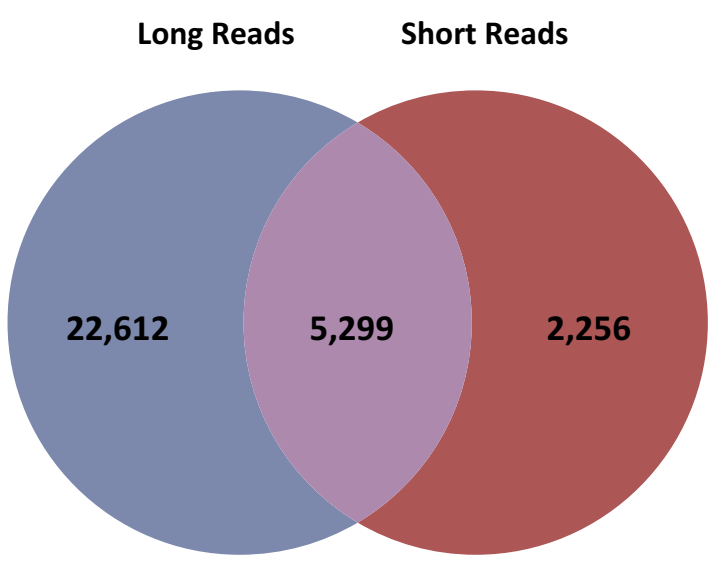

Mouse

Fig. 1 Workflow for circRNA ONT sequencing. a CircRNA enrichment protocol. Total human or mouse brain RNA was subjected to DNase treatment and rRNA depletion before treatment with RNase R to digest the linear RNAs. To further enrich for circRNAs, the remaining linear RNAs were polyadenylated and depleted using poly(A) purification beads. b CircRNA library preparation. The enriched circRNA pool was nicked using an optimized protocol to generate large fragments. After phosphorylation of the $5^{\prime}$ ends of the linearized RNAs, the obtained RNA pool was subjected to polyadenylation to align with the ONT sequencing protocol. c CircRNA panel Long-Read Sequencing (circPanel-LRS). Total RNA was reverse transcribed using a panel of circRNA-specific primers (CSP) extended with an oligo sequence partially overlapping the ONT-cPRM (ONT cDNA primer) forward primer oligo. The second strand of the cDNA was synthesized using a panel of reverse circRNA-specific paired primers extended with an oligo sequence partially overlapping the ONT-cPRM reverse primer oligo. Six PCR cycles were run using the first and second strand cDNA synthesis primer oligos without prior cleanup for the first PCR amplification. After cleaning up the PCR product, the resulting library is amplified using ONT sequencing adjusted and sample indexed primers. Equal molar ratios of the barcoded libraries were mixed and ligated to the ONT sequencing adapter before loading to flowcells for sequencing. d, e Number of circRNAs detected using long- and short-read sequencing in human and mouse brain tissue. 
Table 1 Information on reads that map to circRNAs and the BSJ reads in each dataset.

\begin{tabular}{|c|c|c|c|c|}
\hline & \multicolumn{2}{|l|}{ CircNick-LRS } & \multicolumn{2}{|l|}{ CircPanel-LRS } \\
\hline Filtered reads & $4,273,440$ & $12,683,908$ & $4,005,162$ & $3,954,246$ \\
\hline circRNA mapping reads & $\begin{array}{l}619,770 \text { ( } 14.5 \% \text { of } \\
\text { filtered reads) }\end{array}$ & $\begin{array}{l}4,470,533 \text { ( } 35.3 \% \text { of } \\
\text { filtered reads) }\end{array}$ & $\begin{array}{l}3,922,818 \text { ( } 98 \% \text { of } \\
\text { filtered reads) }\end{array}$ & $\begin{array}{l}3,832,609 \text { ( } 97 \% \text { of } \\
\text { filtered reads) }\end{array}$ \\
\hline CircAtlas circRNAs & 14,698 & 27,501 & 296 & 223 \\
\hline CIRCpedia circRNAs & 12,949 & 19,466 & 199 & 150 \\
\hline Novel circRNAs & 2,851 & 11,380 & 118 & 129 \\
\hline Multi round circRNAs & - & - & 30,009 reads & 45,110 reads \\
\hline
\end{tabular}

a

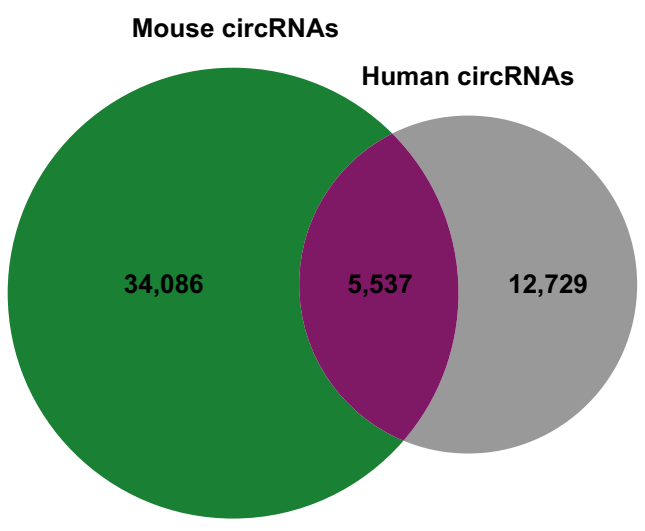

b

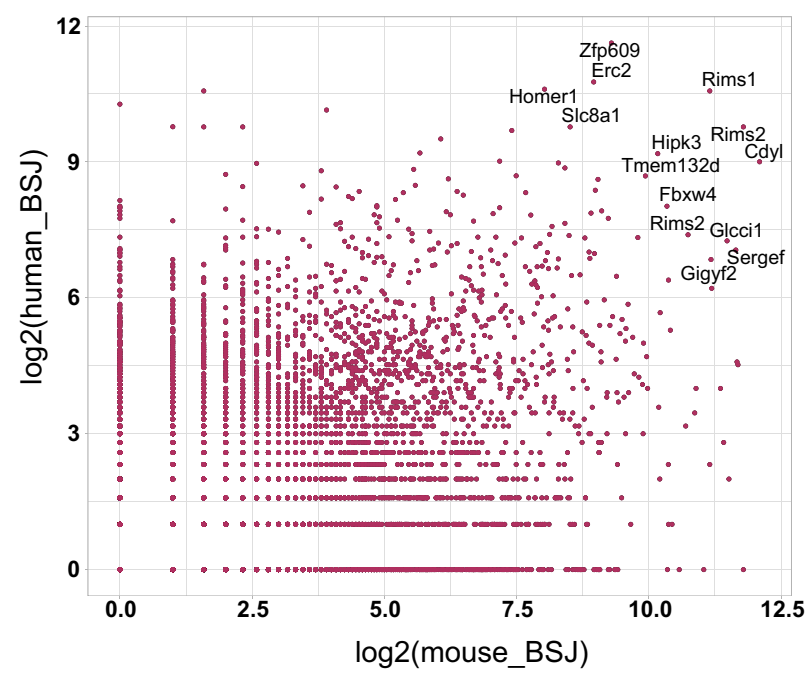

C

KHDRBS3

hg19;

chr8:136,516,672-136,576,635

Human

BSJ long-reads

Linear RNA long-reads

Total RNA short-reads

RefSeq

Conservation

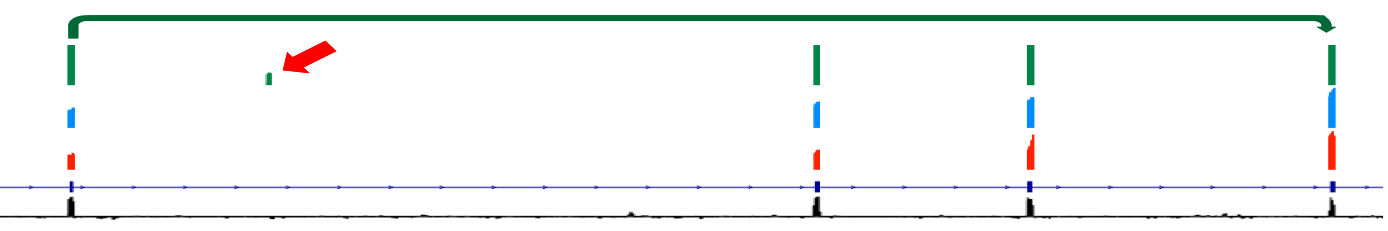

Fig. 2 Conservation between human and mouse brain-derived circRNAs. a Venn diagram showing the total number of circRNAs found in either human $(18,266)$ or mouse $(39,623)$ or in both $(5,537)$. b The number of BSJ-spanning reads detected for mouse ( $x$-axis) vs. human ( $y$-axis) circRNAs. Only circRNAs that are conserved between human and mouse are included. Some genes like Rims1 generate several different circRNAs. c Gene map of human circKHDRBS3, shown as an example where a novel exon (indicated by red arrow) is only detected by ONT sequencing (BSJ long-reads). The green track indicates the BSJ reads in human brain. The light blue track represents reads from full-length linear mRNA sequencing by ONT and the red track indicates short-read sequencing reads for the corresponding sample. The dark blue line refers to RefSeq gene annotation and the black track indicates the level of conservation.

Panel sequencing provides high coverage for specific circRNAs. The circNick-LRS protocol provides an unbiased view of global circRNA expression and allowed us to identify a large number of new circRNAs and visualize circRNA structure; however, the read numbers for many circRNAs were still too low to describe a full isoform composition, particularly for lowly expressed circRNAs.
To get a more detailed view of the alternative splicing landscape of circRNAs, we developed a library preparation strategy that selectively targets a selected set (panel) of circRNAs followed by Long-Read Sequencing (circPanel-LRS). In this approach circRNA-specific primers for cDNA synthesis are annealed to selected regions either $30 \mathrm{nts}$ downstream of the BSJ or the $5^{\prime}$ end 
of a shared exon among different known circRNA variants for the targeted locus (Fig. 1c) to selectively amplify all full-length isoforms carrying this BSJ or the conserved targeted exon. Given the use of circRNA-specific primers, no prior circRNA enrichment step is needed and the amount of input RNA sample can be reduced. We noticed that circPanel-LRS has a tendency to produce concatemeric reads, presumably due to repeated rounds of cDNA synthesis on the circular template enabled by strand displacement activity of the reverse transcriptase (Supplementary Fig. 4).

We tested the circPanel-LRS strategy on total RNA purified from human brain and the neuroblastoma SH-SY5Y cell line using primer sets specific for 10 circRNAs chosen based on variable expression levels, sizes and exon numbers (Supplementary Data 3). Nanopore sequencing of the libraries prepared by circPanel-LRS generated 4,005,162 and 3,954,246 high quality filtered reads for human brain and SH-SY5Y samples, respectively, of which 2,508,109 (62.6\%) and 2,963,459 (75\%) reads crossed the BSJ. In total, we identified 403 and 340 different circRNA variants, respectively, from the 10 chosen loci. Out of these, 118 and 129 were categorized as novel circRNAs (Table 1 and Supplementary Data 4). Supplementary Fig. 5 shows examples of isoforms of circRMST, circHERC1, and circTULP4 detected by circPanel-LRS.

Multi-exon circRNAs display intron retention. To create a comprehensive map of the circRNA splicing landscape, all mouse and human BSJ-spanning reads were aligned to their respective reference genome. To focus our analysis on significant circRNAspecific-intron retention events, only circRNAs with 20 or more BSJ reads and having a mean intronic mapping percentage above $3 \%$ were investigated further. Performing this analysis on the reads obtained using the circNick-LRS protocol we found 1,868 and 4,327 circRNAs in human and mouse, respectively. Of these, 213 and 384 circRNAs contained intron-mapping reads, with 17 and 32 of these containing full-length retained introns in human and mouse, respectively (Supplementary Data 5-6). The circPanel-LRS data showed 25 and 27 circRNA isoforms with intron sequences for human brain and SH-SY5Y cells, respectively, with only one example of conserved intron retention (circRYR2).

Some circRNAs detected by circNick-LRS, such as circREXO4 and circCcar2, contain retained introns but are only expressed in either human or mouse (Fig. 3a, b). CircCAMSAP1 is expressed in both human and mouse but intron retention only occurs in human brain (Fig. 3c, d). Comparing all annotated introns in the circRNA genomic regions, we found that shorter introns are more likely to be retained in circRNAs than longer introns (Fig. 3e, f).

\footnotetext{
Alternative exon usage prevalence in circRNAs. The long-read sequencing data also revealed that alternative exon usage is a widespread phenomenon in circRNAs. We found 559 and 681 circRNA isoforms in the circNick-LRS data for human and mouse, respectively, that contained known or novel exons alternatively used in the circRNA and covered by at least 10 reads (Supplementary Data 7). Examples of alternatively spliced circRNA exons are shown for human circRBM23 and mouse circAtxn1 in Fig. 4a, b. Note that in both examples the alternatively used exons are novel and not annotated in RefSeq. The novel exon in human circRBM23 is expressed to a lower extent compared to neighboring exons and the novel exon in mouse circAtxn1 is circRNA-specific and absent in the linear mRNA.

Performing the same analysis for the 10 circRNA loci in the circPanel-LRS dataset revealed 232 and 165 alternatively used exons in human brain and SH-SY5Y samples, respectively, covered by at least 50 reads (Supplementary Data 8). A large
}

number of alternative isoforms were detected from one locus using circPanel-LRS as illustrated for circRIMS1 in the human brain sample (Supplementary Fig. 6). Interestingly, an analysis of the likelihood of exon inclusion revealed that shorter exons are more likely to be alternatively spliced in human and mouse brains (Fig. 4c-f). Our data also confirmed the existence of two previously described human ciRS-7 isoforms of 1485 and 1301 nts caused by optional intron retention ${ }^{36,37}$.

Detection of novel exons and microexons in circRNA. Approximately $90 \%$ of the exons used in circRNAs are annotated in RefSeq or Gencode, but, notably, $8.5 \%(632)$ and $12.37 \%(1,426)$ of the well-expressed exons ( $>5$ reads) seen in the circNick-LRS data are novel. To further analyze the use of novel exons in circRNAs, we categorized them either as "unique novel exons" (entirely new exons) or "cryptic novel exons" (sharing either the $5^{\prime}$ or $3^{\prime}$ splice sites with the annotated exon; Fig. 5a). Using this annotation, 240 out of 7,430 exons in human and 397 out of 11,520 exons in mouse were detected as unique novel exons and 392 and 1,029 as cryptic novel exons, respectively (all detected with more than 5 reads coverage; Fig. 5b and Supplementary Data 9). CircPanel-LRS, which provides a higher sequencing depth, identified 1,651 and 1,018 exons mapped to the loci of the 10 targeted genes in human brain and SH-SY5Y cells, respectively. Of these, 1,186 and 631 mapped as unique novel exons and 292 and 216 were mapped as cryptic novel exons, respectively (detected with $>5$ reads coverage; Fig. $5 \mathrm{~b}$ and Supplementary Data 10). All single-exon circRNAs are excluded from this analysis since complete coverage of detected exons is required within single reads (see Methods section).

Most novel circRNA exons found in our dataset are in the 40-200 nts range but a distinct group of very short exons of 3-29 nts were also frequently observed, particularly in the unique novel exons group (Fig. $5 \mathrm{c}-\mathrm{j}$ ). These were categorized as microexons in line with previous findings from linear mRNA. Interestingly, the majority of the novel exons detected in circRNAs resulted in frameshifts or incorporation of stop codons (Fig. 6a-h).

By focusing on the microexons covered by at least 5 BSJspanning reads, the circNick-LRS data revealed 53 and 99 unique novel microexons as well as 28 and 59 cryptic novel microexons in human and mouse brain, respectively (Supplementary Data 11 and 12). The circPanel-LRS data, which provides a deeper sequencing coverage for the 10 targeted circRNAs loci, detected 546 and 240 unique novel microexons and 52 and 31 cryptic novel microexons in human brain and SH-SY5Y cells, respectively, with at least 5 BSJ reads (Supplementary Data 13 and 14).

To further analyze the occurrence of well expressed (covered by at least 5 reads) novel microexons in circRNAs, the cryptic- or unique novel exons shorter than $30 \mathrm{nts}$ were divided into two groups (3-15 nts and 16-29 nts). Most unique novel microexons belong to the 3-15 nts group (Fig. 6e, f). CircRNAs are generally not translated and therefore not subjected to nonsense-mediated decay (NMD) if frameshifted. In accordance with this we found $\sim 1 / 3$ in-frame and $2 / 3$ out-of-frame microexons in circRNAs and, within each category, some with at least one stop codon. The circPanel-LRS sequencing showed an even higher number of microexons in the targeted circRNAs, although this may be partly explained by an increased sequencing depth (Fig. $6 \mathrm{~g}$, h). A list of all microexons detected in circRNA and covered by $>10$ reads in circNick-LRS dataset and $>50$ reads in circPanel-LRS are provided as Supplementary Data 15.

We noticed that several of the microexons that are preferentially found in circRNA rather than linear RNA are known for their involvement in neuronal dysregulation 38,39 . In particular, microexons in DTNA (Fig. 7a) and Eif4g3 (Fig. 7b) are mainly found in the circRNA form with $80 \%$ and $32 \%$ inclusion 

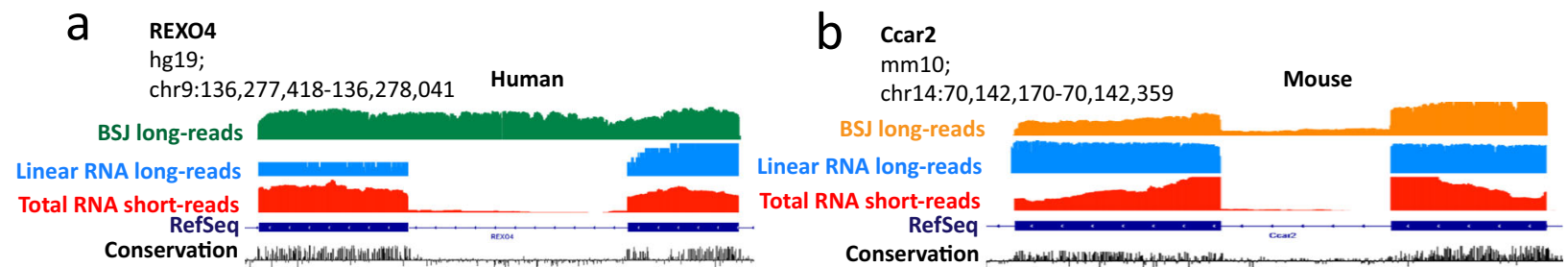

\section{C}

CAMSAP1

hg19;

chr9:138,773,640-138,774,661 Human

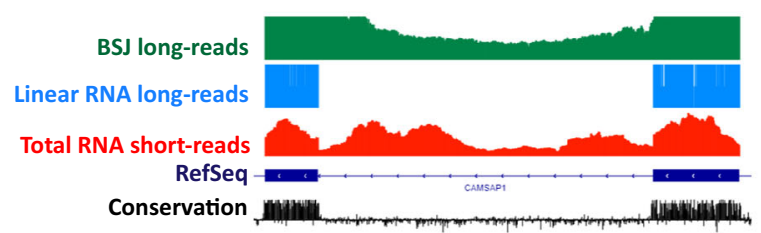

e

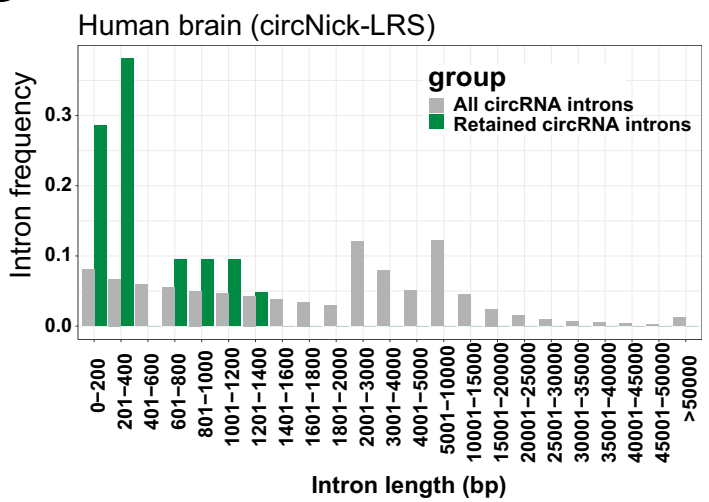

d

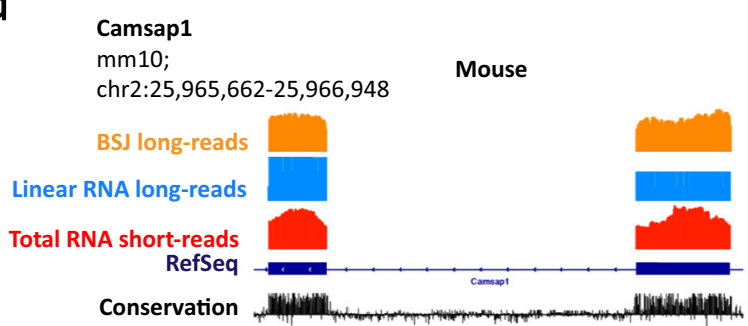

f

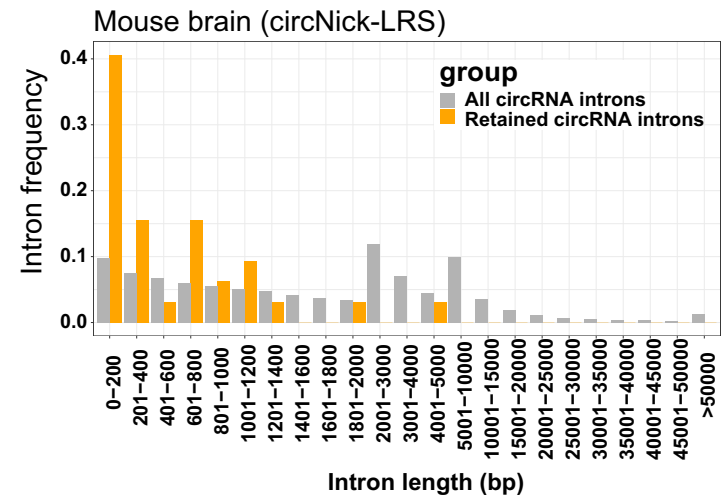

Fig. 3 Examples of intron retention events in circRNAs and their frequency and length distribution. a Human circREXO4 contains a retained intron not detected in linear mRNA. b Mouse CircCcar2 generates different isoforms with retained introns. c, d CircCAMSAP1 is conserved between human and mouse but only the human circRNA shows intron retention. $\mathbf{e}, \mathbf{f}$ The frequency of intron retention in comparison to intron length across all annotated introns in circRNAs genome-wide. Gray bars, frequency of all circRNA introns; green and yellow bars, frequency of retained circRNA introns in human and mouse, respectively. See legend to Fig. 2 for the explanation of tracks.

compared to the neighboring exons, but with $3.5 \%$ and $23 \%$ inclusion in the corresponding linear RNAs, respectively. The circRNA-enriched microexons were validated by RT-PCR for circDTNA (human, Fig. 7c), circEif4g3 (mouse, Fig. 7d) and circDtna (mouse, Fig. 7e) and confirmed by Sanger sequencing (Fig. $7 \mathrm{f}-\mathrm{h}$ ). Other microexons, such as those found in Ank2, Ap1s2, Agrn, Apbb1, and Eif4g3 were found in both circular and linear RNA from the same loci. In the mouse brain, the Asxl3 and Patj genes contain a microexon preferentially included in their respective circular products, while $F n d c 3 b$ contains a microexon exclusively used in circFndc 3 b.

In human brain, ERC2, $A P B B 1$, and $A S A P 1$ show microexon inclusion in both circRNA and linear RNA, while EIF4G3 and ADGRL3 contain microexons mainly found in the circRNA. In addition, TMEM245 and MSANTD3 express microexons that are circRNA-specific and not seen in the linear RNA produced from the same loci.

We compared all microexons in the circNick-LRS dataset $(>10$ BSJ-spanning reads) with the microexons reported by Irimia et al. $^{38}$ as a reference for neuron-specific microexons. For the human brain sample, 31 out of 181 detected microexons and in mouse sample, 19 out of 499 microexons were found in the reference list (Supplementary Data 16).

\section{Discussion}

Delineation of the exact exon composition of mammalian RNAs is often neglected due to predominant use of short-read sequencing. The invention of long-read sequencing provides a unique opportunity to address the question. In this study, we apply ONT longread sequencing technology to describe the exon composition of full-length circRNAs as well as the corresponding mRNA. This approach circumvents the limitation of second-generation pairedend sequencing methods where the read length is limited to $\sim 200$ nucleotides adjacent to the BSJ, insufficient to read through most circRNAs. Previous studies have presented algorithms to predict circRNA structure and alternative splicing events based on shorter sequencing reads ${ }^{16,17}$; however, neither the current secondgeneration sequencing protocol nor prediction algorithms can provide a direct view of the full-length sequence of circRNAs.

A limitation of the ONT sequencing method is the relatively low read number (5-10 million reads compared to 1-2 billion reads for short-read sequencing) and we therefore implemented a circRNA enrichment protocol using successive poly(A)-tailingpoly $(\mathrm{A})$ removal steps. However, one should bear in mind that extensive purification can introduce biases in the circRNA pool. For instance, some linear RNAs are known to be resistant to RNase $\mathrm{R}$ while some circRNAs are sensitive, presumably because 
a

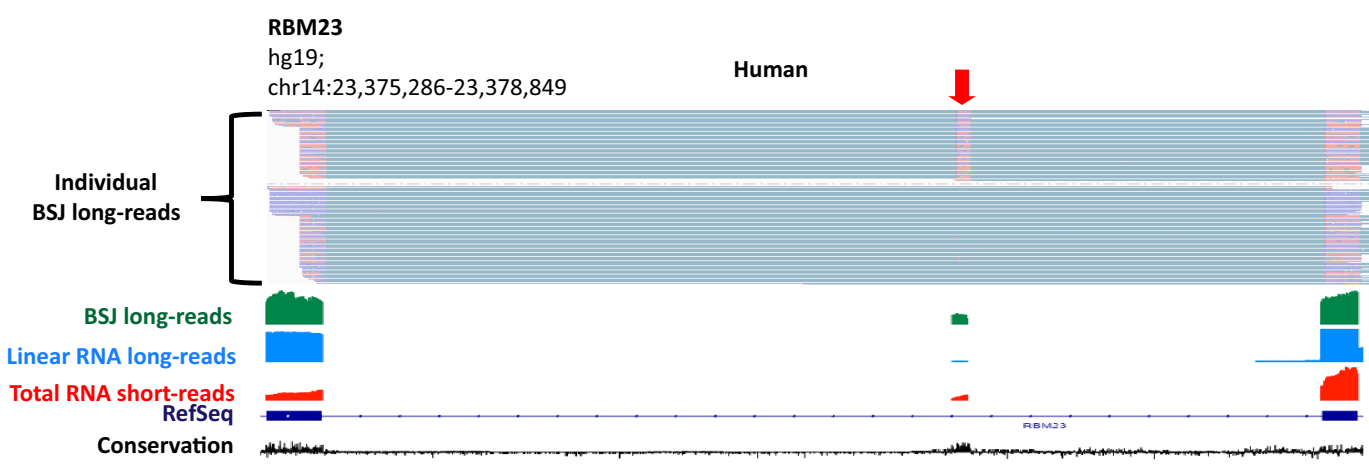

b

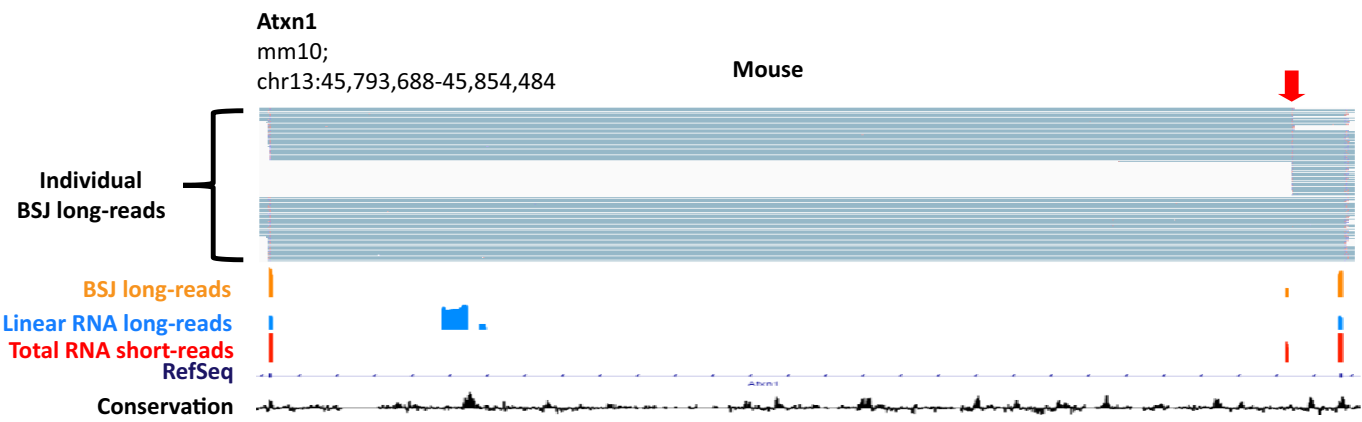

C

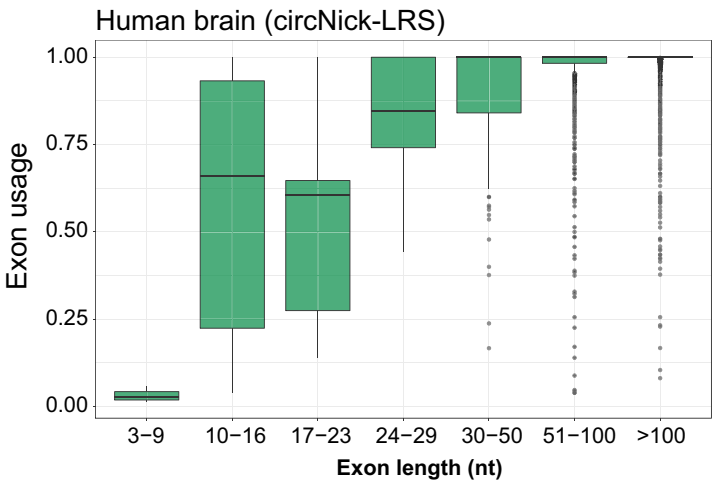

e

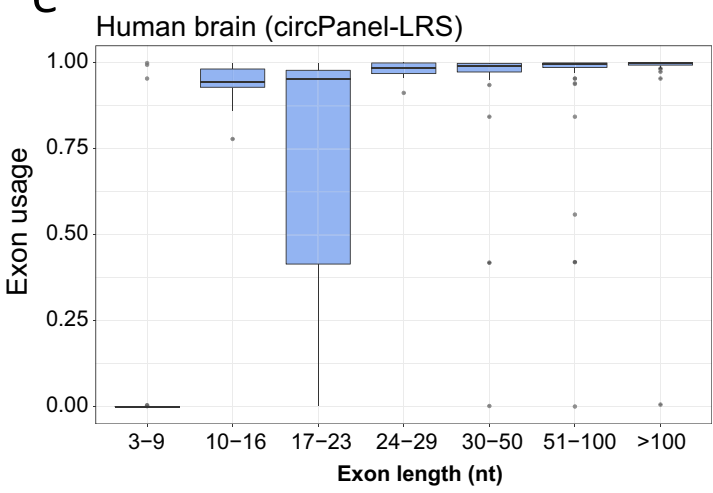

d

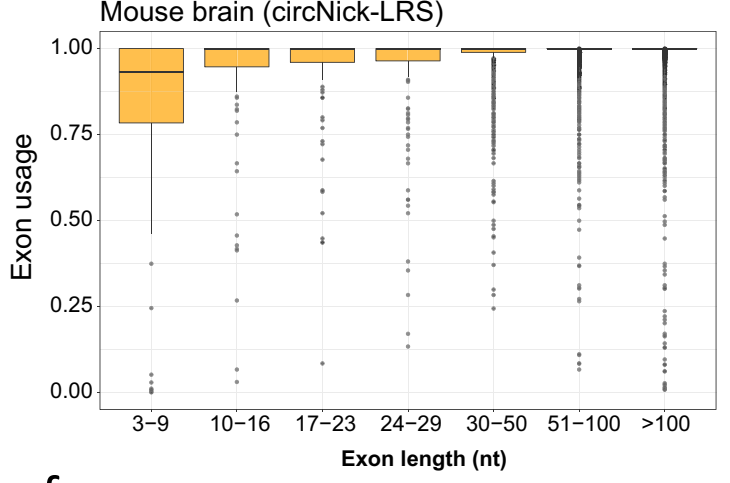

f

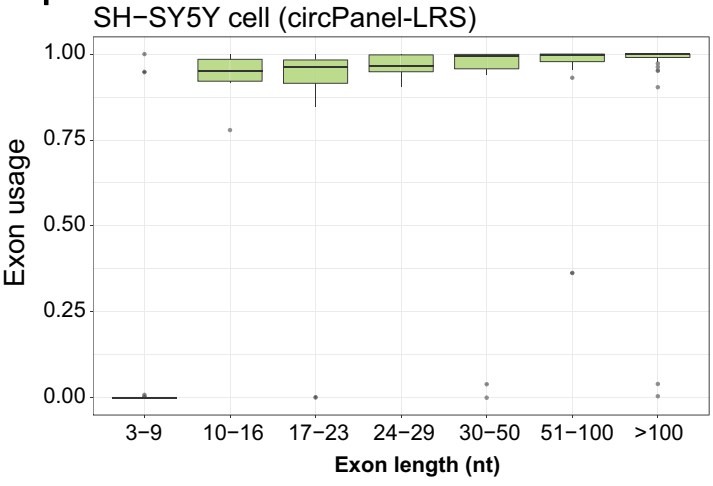

Fig. 4 Examples of circRNA-specific alternative splicing. a Alternative splicing of circRBM23 (red arrow). The alternative exon is primarily included in circRNA. b Example of a circRNA-specific exon in circAtxn1 (red arrow). See legend to Fig. 2 for the explanation of tracks. c-f The level of exon usage for various exon lengths are shown, ranging from no exon usage, 0 on $y$-scale, to constitutive exons, 1 on $y$-scale. Box plot hinges range from the 25th to the 75th percentile, correspond to the first and third quartiles. Horizontal lines indicate median. The upper whisker extends from the hinge to the largest value, but no further than the 75th percentile $+1.5 \times I Q R$, where IQR is the inter-quartile range, or distance between the first and third quartiles. The lower whisker extends from the hinge to the smallest value, but no further than the 25th percentile $-1.5 \times I Q R$. Dots represent exon lengths outside this boundary. Shown for the samples c Human brain (circNick-LRS), d Mouse brain (circNick-LRS), e Human brain (circPanel-LRS), and f SH-SY5Y cells (circPanel-LRS). 


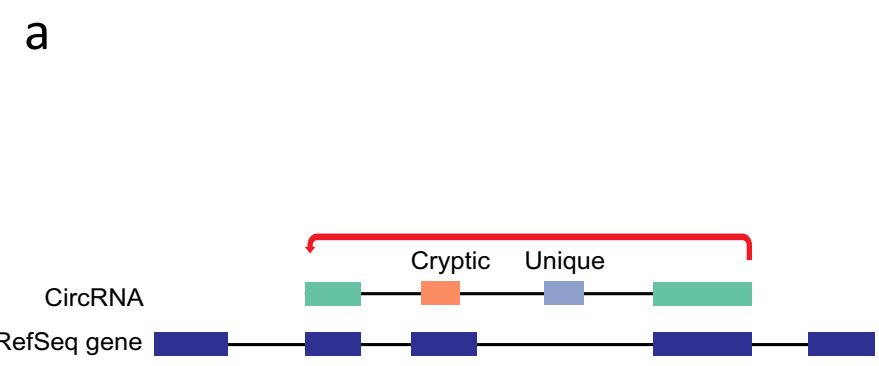

b
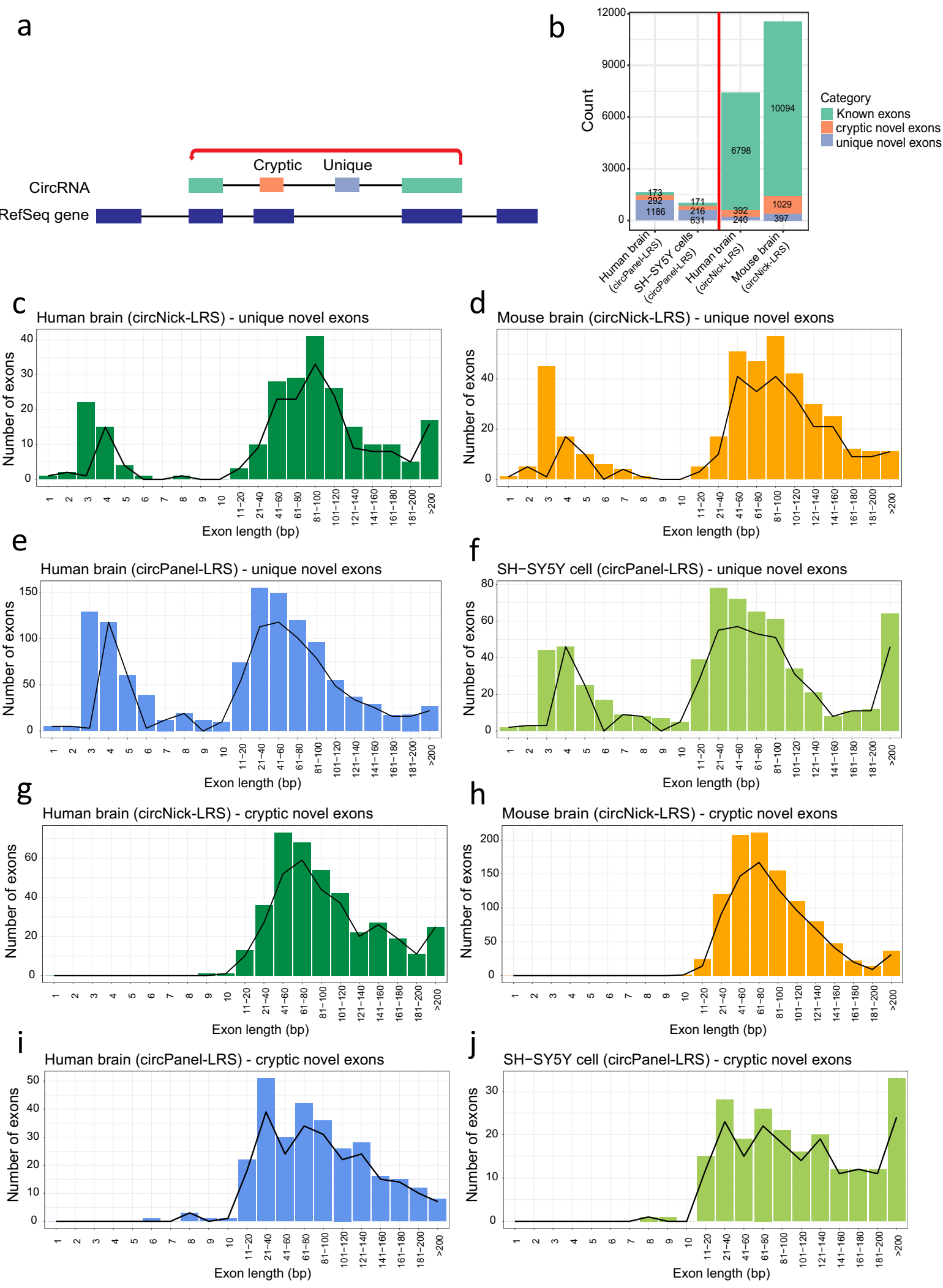

Fig. 5 CircRNAs contain unique and cryptic novel exons. a Schematic representation of circRNAs with novel exons produced from either cryptic spliced exons (peach) or novel exons (blue gray). The arrow indicates the back-splicing event. b Number of known exons, cryptic novel exons, and unique novel exons in circRNAs across all datasets in this study. Only exons covered by at least five long-reads across the back-splice junction are included. $\mathbf{c}-\mathbf{j}$ Distribution of exon length of the unique novel (c-f) and cryptic novel $(\mathbf{g}-\mathbf{j})$ exons in all four datasets made by either circNick-LRS (c, $\mathbf{d}, \mathbf{g}$, and $\mathbf{h})$ or circPanel-LRS (e, $\mathbf{f}, \mathbf{i}$, and $\mathbf{j})$. 
a

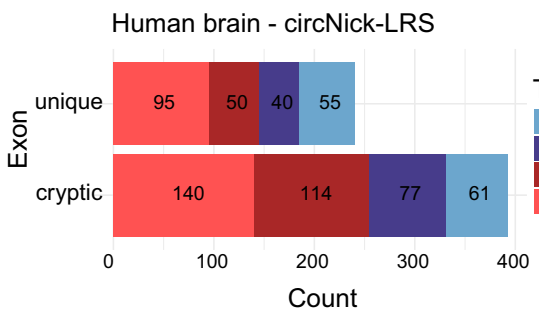

C

Human brain - circPanel-LRS

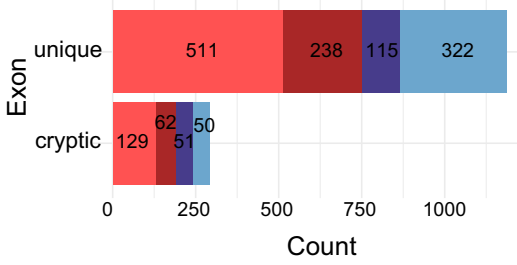

e

Human brain - circNick-LRS

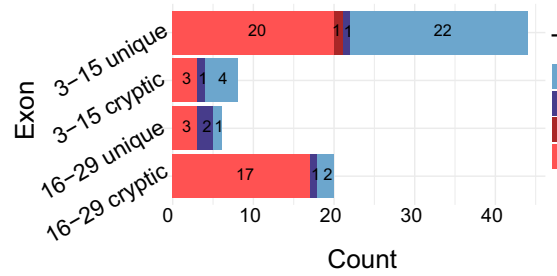

g

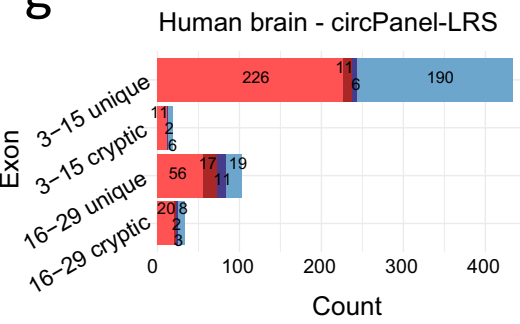

b

Mouse brain - circNick-LRS
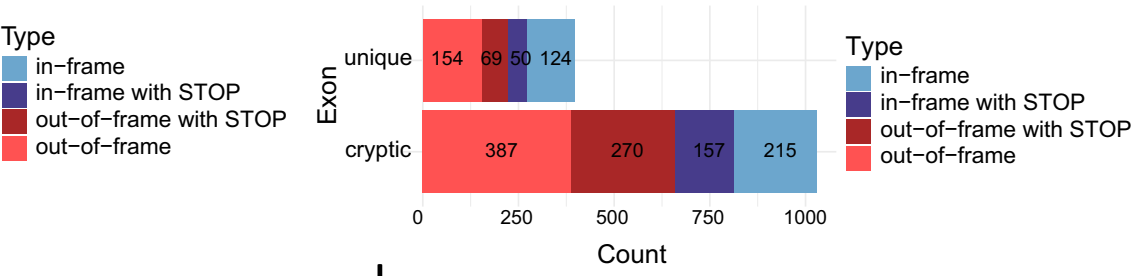

d

SH-SY5Y cell - circPanel-LRS
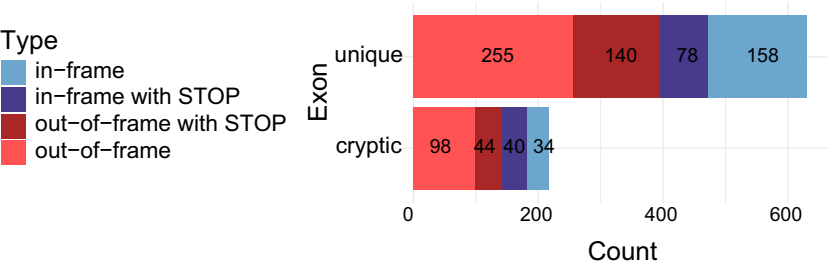

Type
in-frame

in-frame with STOP

out-of-frame with STOP

out-of-frame

f
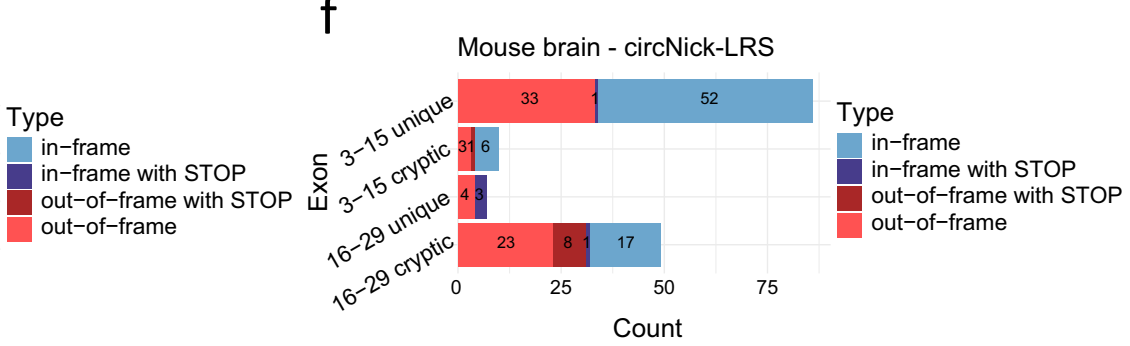

$\mathrm{h}$

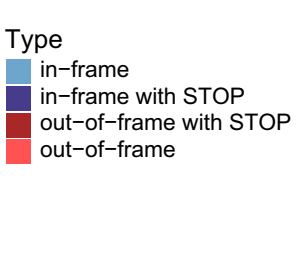

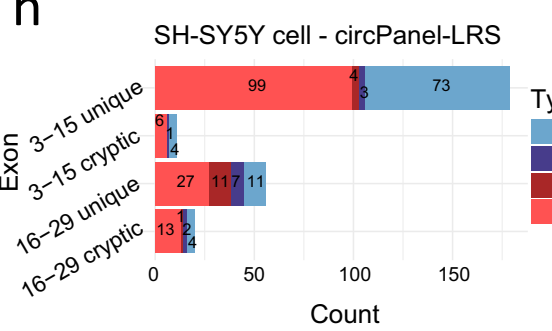

Type

in-frame

in-frame with STOP

out-of-frame with STOP

out-of-frame

Fig. 6 Frequency of frameshifting and stop codons upon inclusion of unique and cryptic novel exons. a-d Exon frame barplots for all unique novel exons (upper bar) and cryptic novel exons (lower bar) in all four datasets. The type of frameshift caused by the circRNA-specific exons are categorized as inframe without stop codon (light blue), in frame with stop codon (dark blue), out of frame without stop codon (light red), and out of frame with stop codon (dark red). e-h Exon frame analysis for all novel microexons (categorized according to length) in all four datasets. All exons are covered by at least 5 long $\mathrm{BSJ}$ reads in this analysis.

they are nicked during purification. In addition, the probes used for rRNA depletion may have coincidental complementarity to circRNAs and lead to their selective removal. Indeed, we found that ciRS-7 was largely depleted in the enriched circRNA pool from mouse while it was covered by $>400$ long BSJ-spanning reads in the human sample. This is likely explained by the presence of several internal poly $(\mathrm{A})$ stretches in ciRS- 7 that can bind the oligo-dT beads used for poly(A) RNA depletion. Indeed we found that circRNA with stretches of 7 or more "A" residues was partially depleted with $33 \%$ and $56 \%$ in the top 1000 detected circRNAs (in the long-reads versus the short-reads datasets) in human and mouse brains, respectively (Supplementary Data 1718). However, we noticed that ciRS-7 levels also vary between human and mouse brain in our short-read sequencing data (511 BSJ reads in human vs $24 \mathrm{BSJ}$ reads in mouse). Mouse ciRS-7 also appeared more sensitive to RNase R, possibly due to its larger size (2927 nts vs $1485 \mathrm{nts}$ in human), as previously observed for other circRNAs $^{32}$.
Our circRNA enrichment protocol furthermore revealed that the BIII-tubulin mRNA is 10-fold more resistant to RNase $\mathrm{R}$ treatment than the Eefla1 mRNA, presumably due to differences in secondary structure. Further investigation is needed to see how RNase R treatment affects different linear and circular RNAs on a global scale. Until then, we need to stress that circRNA purification protocols can create significant biases and that care must be taken to only compare RNA profiles created with the same purification strategy.

To get a deeper view of the alternative splicing landscape of selected, multi-exonic circRNA, we developed circPanel-LRS. This approach does not require circRNA enrichment, making it valuable for low-input samples. The need for gene-specific primers makes this technique ideal for delineating the complexity of circRNAs identified in global screens, from circRNA databases or computational predictions for a region of interest.

The circPanel-LRS method involves an RT enzyme with strand displacement activity and it can therefore generate long-reads that 
derive from multiple 'rounds' of RT activity within the same circRNA (Supplementary Fig. 4). The formation of circRNA concatemers during reverse transcription is a matter of active discussion in the field since it can lead to the overestimation of circRNA levels when using standard quantification methods such as qPCR or short-read RNA sequencing. In contrast, the presence of RT-induced concatemers is readily detected by long-read sequencing.

Our data also enabled a full-length sequence analysis of introncontaining circRNAs known as exon-intron circRNAs (EIciRNAs) in mouse and human brain, some of which were species specific (Supplementary Data 5 and 6 and Fig. 3a, b). These RNAs have been reported to be localized in the nucleus and in some instances increase the expression level of their host gene ${ }^{40}$. This contrasts intron retention in $\mathrm{mRNA}$, which generally represents inefficient splicing and triggers nuclear retention and degradation. However, some retained introns are known to regulate gene expression by nuclear retention ${ }^{41}$ and produce new isoforms with specific functions ${ }^{42}$. Our data do not distinguish nuclear from cytoplasmic circRNA and it remains unclear to what extent intron retention could regulate cellular compartmentalization of circRNA and whether they are associated with regulatory functions.

A large number of alternative splicing events were detected using circNick-LRS and, in particular, when using the higher sequence coverage in circPanel-LRS. Another observation from our long-read sequencing data is the high frequency of unannotated exons found in circRNAs. So why do we observe so many unique and cryptic circRNA exons not normally seen in linear spliced mRNA? One explanation may be that the circRNAspecific exons are also initially spliced into linear mRNA but that the resulting transcripts are more likely to be prone to degradation in the cytoplasm by nonsense-mediated decay (NMD). The NMD pathway is known to target mRNAs carrying a premature termination codon (PTC) in a translation-dependent manner ${ }^{43}$ and, particularly in the brain, NMD is linked to development and neurodegenerative disorders ${ }^{44,45}$. The insertion of noncoding exons between coding exons in linear mRNA is likely to cause NMD, either by shifting the reading frame or by introducing a stop codon. In contrast, since circRNAs are generally not translated ${ }^{46}$, they will not be subjected to NMD and can therefore tolerate the inclusion of novel exons. In support of this theory, more than $76 \%$ and $82 \%$ of novel circRNA-specific exons in mouse and human brain, respectively, are either predicted to introduce frame-shifting or contain at least one inframe stop codon (Fig. 6a-d).

Another possible explanation for the increased occurrence of novel exons in circRNAs could be that the novel exons are only spliced into the circular form of the RNA. The circRNA-specific exons may bind splicing factors or introduce RNA structures that selectively stimulate back splicing events rather than forward splicing. Such a system could function as a proofreading system to correct mRNA splicing by circularizing aberrantly spliced exons. Further investigation is needed to determine whether the alternative circRNA exon structure influences the sub-cellular localization and hence also its potential function. It is also unclear whether circRNA splice-variants are co-expressed in the same cells or originate from different cells in the brain.

Interestingly, a large number of microexons were observed in both human and mouse data (Fig. 6e-h). Microexons generally contain 3-29 nts and are important regulators of the transcriptome, especially in neurogenesis where splicing factors such as nSR100/SRRM4, RBFOX, and PTBP1 regulate the inclusion of brain-specific microexons ${ }^{38,47,48}$. Deeper analysis of our data revealed that some microexons are mainly or exclusively found in circRNAs. In particular, we noticed that several annotated microexons previously linked to autism ${ }^{38,39}$ are preferably found in the circRNA generated from the host gene. Previous studies on microexon annotation relied on short-read RNA sequencing data and therefore misannotated them to linear mRNA transcripts. The observation that several microexons linked to neuronal development and autism are mainly expressed in circRNAs questions their mechanism of action since circRNAs are unlikely to undergo translation ${ }^{46}$. We speculate that the microexons in circRNAs could function as transcription regulators or binding sites for miRNAs and/or proteins ${ }^{49,50}$.

While this study was in revision, two other papers reported the use of nanopore long-read sequencing of circRNAs ${ }^{51,52}$. The isoCirc approach published by Xin et al. initiates cDNA synthesis using random hexamers followed by rolling circle amplification to generate concatemers. Notably, this protocol appears to generate shorter average lengths for the BSJ-containing consensus sequences (300-460 nts) compared to circNick-LRS (average of $795 \mathrm{nts}$ for full-length detected circRNAs in human brain; Supplementary Fig. 7). As an example, circFAT3 (circBase ID: hsa_circ_0000348), a 3,300 nts circRNA highly expressed in the brain, was not detected by isoCirc. Additionally, circPanel-LRS generates full-length cDNA from targeted circRNAs of any size and also makes concatemeric reads from the same circRNA template for better validation. The main difference between isoCirc and circPanel-LRS, in terms of cDNA synthesis and library enrichment, is that circPanel-LRS uses circRNA-specific primers while isoCirc relies on random hexamers. Xin et al. report a relatively high number of new circRNAs isoforms but this may reflect that they only compare their data to circBase and the cancer-specific MiOncoCirc circRNA database whereas we use the more comprehensive databases circAtlas, CIRCpedia, and circBase ${ }^{33-35}$. Furthermore, we note that Xin et al. seem to have misinterpreted our original work on bioRxiv ${ }^{26}$ neglecting that calling a circRNA requires the presence of BSJ-containing reads. Additionally, only circRNA-specific splicing events that meet a defined read coverage of 5,10 , or 20 reads, depending on the type of analysis, were included in our analysis. The relative high number of reads per circRNA allowed us to investigate exon composition, novel exons, alternative exon usage, intron retention, and microexons in thousands of circRNAs.

During the revision of our manuscript, Zhang and colleagues also reported a method for nanopore sequencing of circRNAs called CIRI-long ${ }^{52}$. Here, cDNA synthesis is initiated using random hexamers that amplifies the circRNA signal by forming concatemers of the same circRNA at the RT step and subjecting this to direct cDNA sequencing after library size selection. The average length distribution of the detected full-length circRNAs generated by CIRI-long is $<500 \mathrm{nts}$, which is also shorter than $795 \mathrm{nts}$, the average size of full-length detected circRNAs generated by circNick-LRS approach in human brain sample. Considering the length distribution of the raw reads and the copy number of the concatemeric cDNA sequences, Zhang et al., have mainly covered small circRNAs. Hence, it appears that the shorter reads obtained with both isoCirc and CIRI-long may be a consequence of premature termination caused by annealing random hexamers when an RT enzyme with weak strand displacement and having RNase $\mathrm{H}$ activity is used. In contrast, circNick-LRS relies on circRNA fragmentation, followed by polyadenylation before cDNA synthesis. The fragmentation step in the circNickLRS protocol was optimized to primarily cut once, even in larger circRNAs, to allow full-length circRNA reads. Since larger circRNAs are more likely to be nicked this may create a bias of our method towards longer circRNAs. In addition, we apply a size cut-off in the cDNA amplification step where circRNAs $<200 \mathrm{nts}$ are depleted. These technical biases are avoided in circPanel-LRS where the primer design ensures full-length sequencing of 


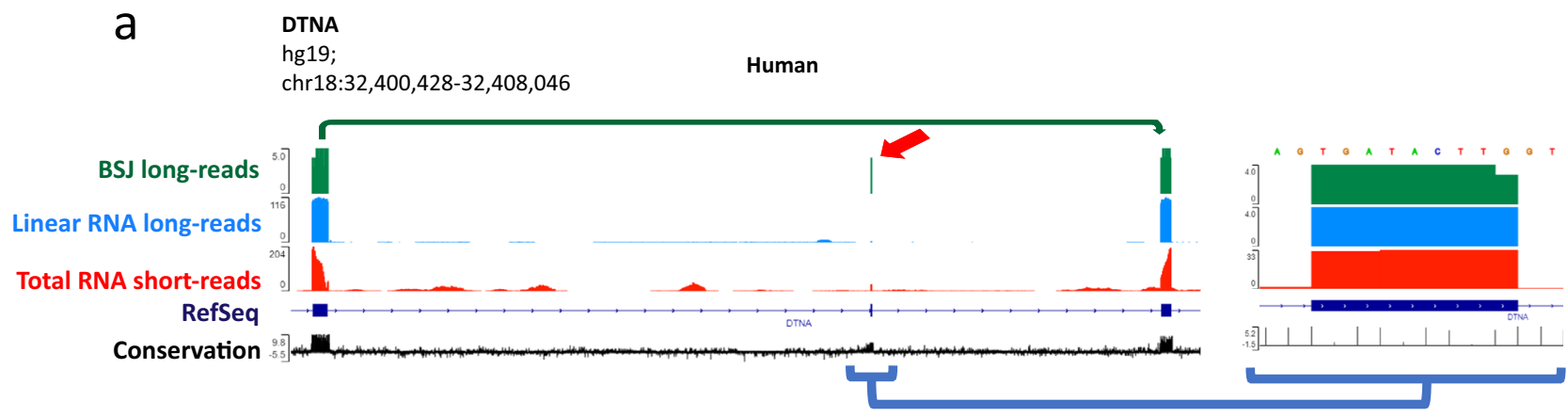

b

\section{Eif4g3}

$\mathrm{mm} 10$;

chr4:138,095,830-138,097,049 Mouse
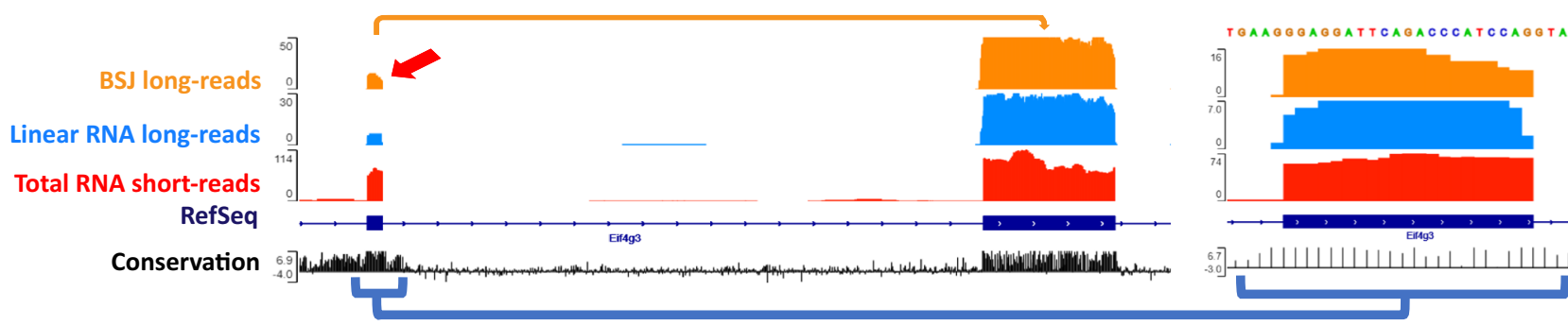

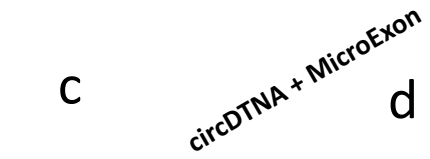

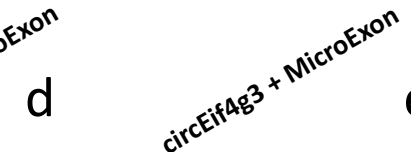

e
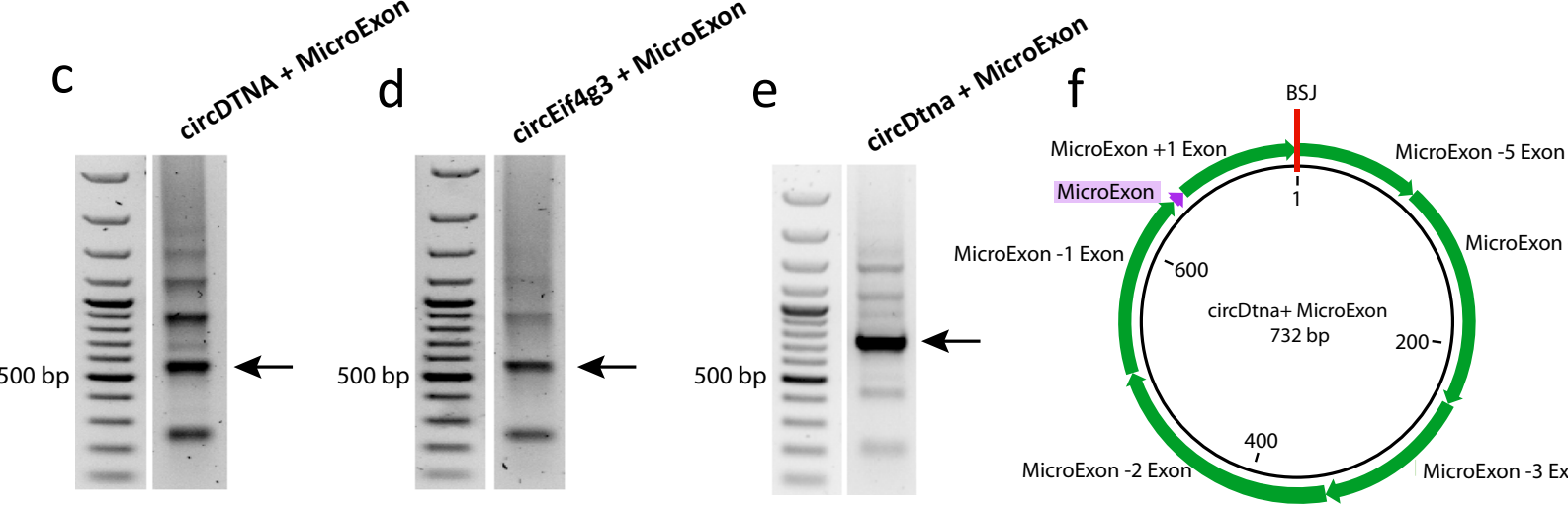

MicroExo

g

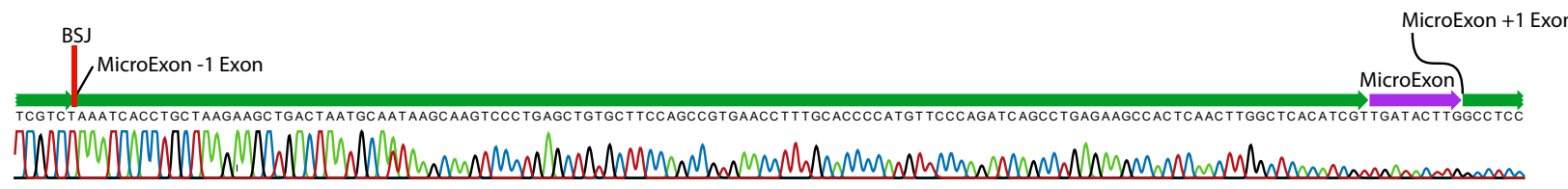

h

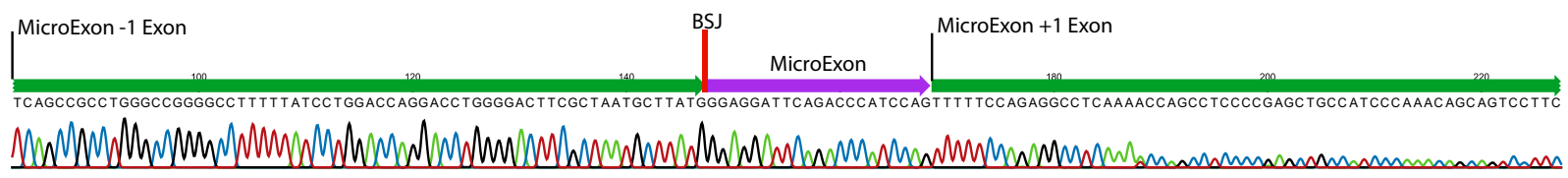

Fig. 7 Examples of microexons in circRNAs detected in BSJ-spanning long-reads. a Microexon located in circDTNA from human brain detected in 4 out of 5 BSJ-spanning reads (80\%; Microexon enlarged to the right). In the long-read linear mRNA, only 4 out of 116 reads (3.5\%) include the microexon with the neighboring exons. This microexon is included in 33 out of 204 reads (16\%) based on short-read sequencing. b Microexon located in circEif4g3 from mouse brain detected in 16 out of 50 BSJ-spanning long-reads (32\%) and 7 out of 30 reads (23\%) in long-read linear mRNA. Short-read sequencing find the microexon included in 74 out of 114 reads (65\%). c-e RT-PCR validation of circRNAs circDTNA (shown in panel a), circEif4g3 (shown in panel b), and circDtna (mouse) using back-to-back primers targeting the exons neighboring the microexons and covering the BSJ. Different bands indicate several circRNA isoforms obtained from the same genomic regions and sharing the same targeted exon. Each RT-PCR has been repeated twice and resulted in the same PCR products. $\mathbf{f}$ Full structure determination of circDtna including the microexon using Sanger sequencing. The circRNA amplicon was gel purified from the PCR band indicated by an arrow in panel e. $\mathbf{g}$, h Microexons in the RT-PCR products (marked by arrows in panels $\mathbf{c}, \mathbf{d}$ for circDTNA and circEif4g3 respectively) were validated by Sanger sequencing; the individual sequencing reads cover both BSJ and the microexon. 
circRNAs without prior circRNA enrichment, something that will be beneficial for labs working with a group of preselected circRNAs.

Importantly, we sequenced both circRNA and linear poly(A) RNA from the same RNA samples, enabling a direct comparison of exon usage in the two groups of RNAs and allowing us to identify and quantify specific splicing events in both types of RNA.

In conclusion, our circRNA enrichment strategy combined with nanopore mediated long-read sequencing allows a detailed characterization of full-length circRNAs in all size ranges. The most striking observation from our study was the large number of circRNA-specific splicing events found in human and mouse brain tissue, which illustrates the importance of long-read sequencing. It remains to be seen whether the unusual splicing pattern of circRNAs merely reflects the absence of NMD or whether alternatively spliced circRNAs mediate new functions independently of the linear host transcript. The latter option is underscored by the notion that several microexons linked to neurodevelopmental disorders are preferentially found in circRNAs, calling for a reevaluation of their mechanism of action.

\section{Methods}

In order to cover the circular RNAs derived from both X and Y chromosomes, total RNA was obtained from male gender for both human and mouse samples. Total human brain RNA was derived from post mortem cortex and provided by Agilent (Agilent Technologies, cat: 540143). For the preparation of mouse brain RNA, a 2 months old male mouse (strain 129S2/SV) was killed and the entire brain was harvested and grained in Trizol (Thermo Fisher Scientific). Total RNA was obtained according to the manufacture's protocol. Animals were treated according to the regulation of "The Animal Experiments Inspectorate", the legal authority under the "Ministry of Environment and Food of Denmark" (https://www. foedevarestyrelsen.dk/english/Animal/AnimalWelfare/The-Animal-ExperimentsInspectorate/Pages/defaultaspx). All mice had free access to food and water supplied ad libitum and were kept under 14 -h light/10-h dark cycle and standard conditions of ambient temperature $\left(22^{\circ} \mathrm{C} \pm 1{ }^{\circ} \mathrm{C}\right)$ and humidity $(50 \% \pm 10 \%)$.

DNA LoBind tubes (Eppendorf) were used for all steps during the RNA preparation and ONT cDNA-PCR library construction. RNA and dsDNA concentrations were determined using a Qubit 4.0 fluorometer together with the Qubit HS dsDNA and Qubit HS RNA kits (Thermo Fisher Scientific). RNA quality was assessed after each step of circRNA preparation and polyadenylation using Agilent 2100 bioanalyzer (Agilent Technologies). DNase I treatment was utilized to remove DNA from both the human and mouse total RNA preparations. The quality of total RNA samples was confirmed by agarose gel analysis and Agilent 2100 bioanalyzer.

Total RNA short-read sequencing. Human and mouse brain samples were used for short-read total RNA sequencing. The rRNA was depleted and sequencing was performed using DNA nanoball sequencing technology and BGISEQ-500 sequencing platform and 30 million paired-ends (PE100) reads were generated per sample.

\footnotetext{
Preparation of linearized polyadenylated circRNA for nanopore sequencing Generally, RNA Clean and Concentrator ${ }^{\mathrm{TM}}-5$ (R1016, Zymo Research) was applied after each step in the RNA preparation procedure to clean up and concentrate the RNA, using the adjusted RNA Binding Buffer to select only RNA transcripts longer than 200 nts. RiboLock RNase Inhibitor (Thermo Fisher Scientific, EO0381) was added to the eluted RNA to prevent RNA degradation after each step. First, the Ribo-Zero rRNA Removal Kit (Human/Mouse/Rat, Illumina) was employed to deplete ribosomal RNA from $20 \mu \mathrm{g}$ of total human or mouse brain RNA according to the manufacture's instruction. Then, the rRNA-depleted sample was treated with RNase R (Epicentre, RNR07250) to digest all linear RNAs which are not resistant to degradation by RNase R. In order to further enrich the ratio of circRNAs to linear RNA, the remaining linear RNAs were polyadenylated utilizing the Poly(A) Polymerase (New England Biolabs, M0276) according to the manufacturer's instruction and subsequently removed with NEBNext Poly(A) mRNA Magnetic Isolation Module (New England Biolabs, E7490S) following the kit's manual. Supernatant obtained from the first step of the poly(A) purification contained the Enriched CircRNA Pool (ECP) and was used in the next step.

Next, a NEBNext Magnesium RNA Fragmentation Module (New England Biolabs, E6150) was used to linearize the circRNAs obtained from ECP and in preparation for sequencing. In order to fragment both small and large circRNAs, and prevent over-degradation of large circRNAs, the ECP sample was divided into three aliquots and subjected to $80^{\circ} \mathrm{C}$ incubation for $30 \mathrm{~s}, 1$ or $2 \mathrm{~min}$, respectively, before pooling them again. After fragmentation, the 3'phosphate group was
} removed from linearized circRNAs, and phosphate groups added to the 5 'ends using T4 Polynucleotide Kinase (New England Biolabs, M0201S) by first incubating for $30 \mathrm{~min}$ without ATP and then $30 \mathrm{~min}$ with ATP to remove and add the phosphate group, respectively. Finally, the linearized RNAs were polyadenylated as described above and used as input for the cDNA-PCR sequencing procedure (Fig. 1a, b). We named this strategy circRNA Nicking and Long-Read Sequencing (circNick-LRS).

Evaluation of circRNA abundance during RNA preparation. For cDNA preparation and to evaluate the efficiency of circRNA enrichment in ECP and for microexon validation by RT-PCR, we used the Superscript VILO cDNA Synthesis Kit (Thermo Fisher Scientific) according to the manufacturer's protocol. The LightCycler 480 SYBR Green I Master Kit was used for the qPCR reactions. $\beta I I I-$ tubulin was chosen as housekeeping gene, $18 \mathrm{~S}$ ribosomal RNA as a marker for rRNAs and circHipk 3 to represent the circRNA. To do RT-PCR for microexon validation, LongAmp Hot Start Taq 2X Master Mix (New England Biolabs, M0533L) was used following the manufacturer's instruction. Sequence of the primers provided in Supplementary Table 1.

CircNick-LRS CDNA-PCR sequencing. The manual of cDNA-PCR Sequencing Kit (SQK-PCS108, version PCS_9035_v108_revF_26Jun2017), provided by Oxford Nanopore was applied with a few modifications as described below.

Reverse transcription and strand-switching. Briefly, $9 \mu$, equal to $9 \mathrm{ng}$ of polyadenylated ECP RNA, $1 \mu \mathrm{l}$ VNP primer (ONT), and $1 \mu \mathrm{l} 10 \mathrm{mM}$ dNTPs were mixed and incubated at $65^{\circ} \mathrm{C}$ for $5 \mathrm{~min}$ and snap-cooled on a pre-chilled freezer block. Then, a mix of $4 \mu$ l Superscript IV buffer (Thermo Fisher Scientific), $1 \mu l$ RNaseOUT, $1 \mu \mathrm{l} 100 \mathrm{mM}$ DTT, and $2 \mu \mathrm{l}$ Strand-Switching Primer (SSP, ONT) was added to the cold sample, followed by incubation at $42^{\circ} \mathrm{C}$ for $2 \mathrm{~min}$. Finally, $1 \mu \mathrm{l}$ of $200 \mathrm{U} / \mu \mathrm{l}$ Superscript IV Reverse Transcriptase (Thermo Fisher Scientific) was added and the following protocol completed using a thermocycler: One cycle at $50^{\circ} \mathrm{C}$ for $10 \mathrm{~min}$ (reverse transcription), one cycle at $42^{\circ} \mathrm{C}$ for $10 \mathrm{~min}$ (strandswitching), one cycle at $80^{\circ} \mathrm{C}$ for $10 \mathrm{~min}$ (heat-inactivation) and cool down to $4{ }^{\circ} \mathrm{C}$

PCR amplification of CDNA. A set of $4 \times 50 \mu$ reactions were prepared, each containing $25 \mu \mathrm{l}$ LongAmp Taq 2X Master Mix (New England Biolabs, M0287S), $3 \mu \mathrm{l}$ cDNA PRM primer (cPRM, ONT), $17 \mu \mathrm{l}$ nuclease-free water, and $5 \mu \mathrm{l}$ reversetranscribed RNA sample. The cycle steps of the PCR were: (1) $95^{\circ} \mathrm{C}$ for $30 \mathrm{sec},(2)$ $95^{\circ} \mathrm{C}$ for $15 \mathrm{sec}$, (3) $62^{\circ} \mathrm{C}$ for $15 \mathrm{sec}$, (4) $65^{\circ} \mathrm{C}$ for $6 \mathrm{~min}$, repeat steps $2-4$ for $18 \mathrm{X}$, (5) $65^{\circ} \mathrm{C}$ for $6 \mathrm{~min}$ and (6) cool down to $4^{\circ} \mathrm{C}$. Each PCR reaction was treated with $1 \mu \mathrm{l}$ of Exonuclease I (New England Biolabs, M0293S) at $37^{\circ} \mathrm{C}$ for $15 \mathrm{~min}$ followed by $80^{\circ} \mathrm{C}$ for $15 \mathrm{~min}$ to inactivate the Exonuclease I enzyme.

Agarose gel purification and size selection of the PCR products. A set of $4 \times$ $40 \mu \mathrm{l}$ of PCR reaction was mixed with $8 \mu \mathrm{l} 6 \mathrm{X}$ DNA load dye (Thermo Fisher Scientific) and run on a $\%$ TBE agarose gel (UltraPure, Thermo Fisher Scientific) for $2 \mathrm{~h}$ at $80 \mathrm{~V}$. Using the $100 \mathrm{bp}$ and $1 \mathrm{~kb}$ DNA Quick-Load ladders (New England Biolabs, N0467 and N0468) as a reference, the PCR products in the range from $350 \mathrm{bp}$ to $10 \mathrm{~kb}$ were cut out and extracted from the gel (GeneJET Gel Extraction Kit K0691, Thermo Fisher Scientific).

Re-amplification of the gel-extracted library. The $350 \mathrm{bp}-10 \mathrm{~kb}$ products were subjected to PCR amplification and Exonuclease I digestion as described above, except that $2 \mu \mathrm{l}$ cPRM and 6 cycles of steps $2-4$ was applied. In order to confirm the quality of amplified DNA, $5 \mu$ of the PCR product was analyzed in a $2 \%$ TBE agarose gel. The remaining $\sim 200 \mu \mathrm{l}$ PCR material was mixed with $160 \mu \mathrm{l}$ AMPure XP beads (Beckman Coulter) followed by rotation for $5 \mathrm{~min}$ at room temperature. The beads were subsequently washed twice using $500 \mu \mathrm{l}$ fresh $70 \%$ ethanol, and DNA material eluted from the beads using $21 \mu \mathrm{l}$ of Rapid Annealing Buffer (RAB, ONT) during $10 \mathrm{~min}$ rotation at room temperature. To add the adapter, $400 \mathrm{fmol}$ of the eluted DNA was adjusted to $23 \mu \mathrm{l}$ using RAB buffer and used for adapter ligation by addition of $2 \mu \mathrm{l} \mathrm{cDNA}$ adapter mix (cAMX, ONT) and incubating for $5 \mathrm{~min}$ at room temperature with rotation. The mixture was purified using $20 \mu \mathrm{l}$ of AMPure XP beads, incubated for $5 \mathrm{~min}$ at room temperature with rotation, followed by washing the beads twice with $140 \mu$ lof Adapter Bead Binding Buffer (ABB, ONT). Elution was done with $13 \mu \mathrm{l}$ of elution buffer (ELB, ONT) for $10 \mathrm{~min}$ at room temperature with rotation. Then $12 \mu \mathrm{l}$ of the ELB eluate was mixed with $35 \mu \mathrm{l}$ of Running Buffer with Fuel mix (RBF, ONT), $25.5 \mu \mathrm{l}$ of Library Loading Beads (LLB, ONT), and $2.5 \mu$ of nuclease-free water, and the sample sequenced for $48 \mathrm{~h}$ using the MinION Mk1B with a FLO-MIN106 R9 Flow Cell.

CircRNA Panel Long-Read Sequencing (circPanel-LRS). A more efficient and convenient approach was developed for long-read and multiplex sequencing and splicing events analysis of an already selected panel of circRNAs (circPanel-LRS). The circPanel-LRS protocol was adapted to target and sequence a panel of selected circRNAs directly using total RNA without need of any prior circRNA enrichment and with the possibility of multiplexing different samples combined and sequenced in one round. 
A panel of 10 circRNAs was selected based on sequencing data obtained from the circNick-LRS long-read approach and also short-reads data from the human brain sample. The circRNAs were selected from different sizes in length and from low to high expression (Supplementary Data 3). As shown in Fig. 1c, 25 nts length primers were designed for reverse transcription to anneal either $30 \mathrm{nts}$ down of the BSJ or $5^{\prime}$ end of the mostly shared exon, covering as many as possible different circRNA isoforms obtained from the same host linear. Primers were designed at least with $40 \% \mathrm{GC}$ and Tm of more than $60^{\circ} \mathrm{C}$ in the LongAmp Hot Start Taq 2X Master Mix buffer (New England Biolabs, M0533L). These primers were extended at the $5^{\prime}$ end to be targeted by PCR amplification and samples barcoding primers and to be adjusted to the ONT CDNA-PCR sequencing protocol with (SQKPCB109) or without (SQK-PCS109) sample barcoding (Supplementary Data 3).

For cDNA synthesis, Maxima H Minus Reverse Transcriptase (Thermo Fisher Scientific, EP0752) was used and a total of 10 preselected circRNAs loci were targeted. Briefly, $1 \mu \mathrm{g}$ of DNase I treated total RNA, $1 \mu \mathrm{l}$ of a 10 pmol primer mix (pool of 10 primers containing 1 pmol of each primer), and $1 \mu \mathrm{l} 10 \mathrm{mM}$ dNTPs ( $2.5 \mathrm{mM}$ each) pooled and up to $14 \mu \mathrm{l}$ nuclease-free water was added. RNA-Primer mix was incubated at $70^{\circ} \mathrm{C}$ for $3 \mathrm{~min}$ and snap cooled down on ice. Later, $4 \mu \mathrm{l} 5 \mathrm{x}$ RT Buffer for Maxima H Minus Reverse Transcriptase (Thermo Fisher Scientific) and $1 \mu$ l RiboLock RNase inhibitor $(40 \mathrm{U} / \mu \mathrm{l})$ were added and incubated at $42{ }^{\circ} \mathrm{C}$ for $2 \mathrm{~min}$. Gently, $100 \mathrm{U}$ Maxima $\mathrm{H}$ Minus RT enzyme was added and incubated for $30 \mathrm{~min}$ at $50^{\circ} \mathrm{C}$, followed by $5 \mathrm{~min}$ at $85^{\circ} \mathrm{C}$ to inactivate the RT enzyme and kept at $4^{\circ} \mathrm{C}$. Afterward, $1 \mu \mathrm{l}$ RNase H (Thermo Fisher Scientific, EN0201) and $1 \mu$ l RNase Cocktail Enzyme Mix (Thermo Fisher Scientific, AM2286) were added and incubated at $37^{\circ} \mathrm{C}$ for $20 \mathrm{~min}$ and followed by $20 \mathrm{~min}$ at $75^{\circ} \mathrm{C}$, then kept at $4{ }^{\circ} \mathrm{C}$. The second strand of the cDNA was made using back-to-back panel circRNA-specific divergent primers. The final $22 \mu \mathrm{l}$ cDNA of each sample from previous step, was divided into two $0.2 \mathrm{ml}$ PCR tubes and the following materials were added to each tube separately including $25 \mu \mathrm{l}$ LongAmp Hot Start Taq 2X Master Mix (New England Biolabs, M0533L), $1 \mu$ l of the $10 \mathrm{pmol}$ second strand cDNA synthesis primer mix (1 pmol of each primer) and $13 \mu \mathrm{l}$ nuclease-free water added up to the final of $50 \mu \mathrm{l}$. In order to synthesize the second strand of the CDNA and to enrich the targeted circRNA isoforms, the PCR reactions prepared in the previous step were incubated for 1 cycle of $2 \mathrm{~min}$ at $95^{\circ} \mathrm{C}, 1 \mathrm{~min}$ at $50^{\circ} \mathrm{C}$, and $10 \mathrm{~min}$ at $65^{\circ} \mathrm{C}$, followed by 2 cycles of (1) $95^{\circ} \mathrm{C}$ for $15 \mathrm{sec}$, (2) $55^{\circ} \mathrm{C}$ for $15 \mathrm{sec}$, (3) $65^{\circ} \mathrm{C}$ for $5 \mathrm{~min}$, and followed by 6 more cycles of (1) $95^{\circ} \mathrm{C}$ for $15 \mathrm{sec}$, (2) $62^{\circ} \mathrm{C}$ for $15 \mathrm{sec}$, (3) $65^{\circ} \mathrm{C}$ for $5 \mathrm{~min}$, and one cycle of $5 \mathrm{~min}$ incubation at $65^{\circ} \mathrm{C}$ and cool down to $4{ }^{\circ} \mathrm{C}$

Each PCR reaction was treated with $1 \mu \mathrm{l}$ of Exonuclease I (New England Biolabs, M0293S) and incubated at $37^{\circ} \mathrm{C}$ for $15 \mathrm{~min}$ to digest any unincorporated primers and incubated at $80^{\circ} \mathrm{C}$ for $15 \mathrm{~min}$. The PCR products were cleaned up using SPRIselect beads and the library was subjected to 14 cycles of PCR using barcoded and ONT-adjusted primers (cPRM primers) from the SQK-PCB109 kit. Briefly, the total of $100 \mu \mathrm{l}$ cDNA-PCR product per sample pooled and $65 \mu \mathrm{l}$ well-mixed SPRISelect beads was added $(0.65 \mathrm{X})$ to each tube, mixed and rotated for $10 \mathrm{~min}$ at room temperature. Later, the beads were pelleted and washed twice using $500 \mu \mathrm{l}$ freshly prepared $80 \%$ Ethanol. The pellet was dried for $2.5 \mathrm{~min}$, resuspended in $25 \mu \mathrm{l}$ nuclease-free water by pipetting and incubated for $5 \mathrm{~min}$ at $37^{\circ} \mathrm{C}$ followed by $5 \mathrm{~min}$ rotation at room temperature. The beads were pelleted for $5 \mathrm{~min}$ on a magnetic rack and the supernatant containing the purified library was harvested. Later, the second round of the PCR was applied using barcoded cPRM primers from the ONT SQK-PCB109 kit. For each sample $4 \times 0.2 \mathrm{ml}$ PCR tubes were prepared each containing $2 \mathrm{ng}$ cDNA library from the previous PCR, $25 \mu \mathrm{l}$ LongAmp Hot Start Taq 2X Master Mix (New England Biolabs, M0533L), $1.5 \mu \mathrm{l}$ cDNA PRM primer (cPRM, ONT) provided for SQK-PCB109 kit, and nucleasefree water was added up to $50 \mu$ l. The PCR reactions were incubated for 1 cycle of 2 min at $95^{\circ} \mathrm{C}$, followed by 14 cycles of (1) $95^{\circ} \mathrm{C}$ for $15 \mathrm{sec}$, (2) $62^{\circ} \mathrm{C}$ for $15 \mathrm{sec}$, (3) $65^{\circ} \mathrm{C}$ for $5 \mathrm{~min}$, and one cycle of $5 \mathrm{~min}$ incubation at $65^{\circ} \mathrm{C}$ and kept at $4{ }^{\circ} \mathrm{C}$. Exonuclease I treatment was applied and the PCR products were cleaned up using SPRISelect beads as explained for the first PCR round, except that the library was eluted in $15 \mu \mathrm{l}$ elution buffer (EB, provided by ONT SQK-PCB109 kit). Quantity, quality, and purity of the libraries were assessed using Qubit 4, Agilent 2100 bioanalyzer, and Nanodrop respectively.

A mix of an equimolar ratio of libraries were pooled and multiplexed up to a total of $100 \mathrm{fmol}$ and used for ONT sequencing adapter ligation and ONT MinION sequencing following the manufacturer's instruction for the SQK-PCB109 kit.

Data analysis. Nanopore data was basecalled using Guppy (v 3.4.5), which included barcode trimming. Fastq files produced by Guppy were filtered using NanoFilt (v 2.6.0) allowing only reads with average base quality of 7 or more and a read length of $250 \mathrm{bp}$ or more. The filtered data was mapped to the human genome (hg19) or mouse genome (mm10) using a parallelized version of Blat software package ${ }^{53,54}$. Blat is able to map sequences across linear splice events but when encountering a BSJ splicing event, it splits into two segments that individually can be mapped to the genome. The observation of two segments that map upstream of a splice donor and downstream of a splice acceptor, respectively, signifies a BSJ and can hence be interpreted as a circRNA. A Blat score is calculated as the number of matching nucleotides subtracting mismatches and gap penalties. To qualify as a circRNA, the Blat score has to reach 30 on both sides of the BSJ. A blat score of 30 is a stringent requirement, so only a single read fulfilling this criterium is required to define a circRNA in this study. A putative circRNA is reported if the following criteria are met: the two hits from the same read must be (1) on the same genomic strand, (2) within $1 \mathrm{Mb}$, (3) must not overlap by 50 or more bp, and (4) must be oriented in reverse order relative to the read sequence.

Reads that fulfill the above-mentioned criteria are annotated with the number of bp overlapping a refSeq gene, exon of refSeq gene, Expressed sequence tags (ESTs) from the UCSC genome browser and introns of refSeq genes. This information is stored for each single read and is used later to assess the intron usage level in each detected circRNA. For visualization and further analysis, psl files were converted to bed12 format using a custom script and to bam format using samtools.

Using custom scripts, hits originating from the same read were combined, only allowing reads with exactly two hits marking the genomic ends of circRNAs. The previously added gene/exon/EST/intron bp annotation data is summed when read hits are combined. Reads mapping to mitochondria and rRNA genes are excluded from this analysis. Since the ends of these putative circRNA reads often are heterogeneous due to the data quality of the Nanopore $1 \mathrm{D}$ reads, end-processing of the reads is performed by searching for the closest refSeq exon using bedtools. If both ends of a putative circRNA are within $30 \mathrm{bp}$ of an annotated refSeq exon, the end-sequences of the putative exon are corrected accordingly. This effectively removes the end heterogeneity of the Nanopore data, when reads originate from RefSeq annotated genes. Putative circRNAs that do not satisfy this requirement are matched against circRNAs annotated on circBase, circAtlas, and CIRCpedia. If a read has $95 \%$ overlap in its genome mapping position to an annotated circRNA, the end-sequences of the putative circRNA are corrected to fit the annotated circRNA ends. All reads satisfying the two adjustment criteria are accepted as circRNA candidates.

CircRNA candidate reads are collapsed to show only one unique genomic region for each position found to produce a circRNA. These unique circRNA regions are annotated with overlapping gene (host gene), circBase ID, circAtlas ID and CIRCpedia ID where available, the count of candidate reads that map to the unique region, as well as the mean value of the previous gene/exon/EST/intron bp annotations for the mapping reads. Finally, the minimum, maximum, and mean number of bp the mapping reads were adjusted to fit the mapping circRNA genomic region is added.

Fastq quality scores are defined as $Q=-10 \times \log ($ err), where err is the per base error-rate. The error-rate can be calculated as: err $=10^{\wedge}(-Q / 10)$. Example for quality score of 11.95 : err $=10^{\wedge}(-11.95 / 10)=0.064=6,4 \%$. NanoPlot $(\mathrm{v} 1.29 .0)$ was used to visualize read length and quality.

Custom perl and bash scripts can be found on github (https://github.com/ omiics-dk/long_read_circRNA).

Conservation of circRNAs. Conservation of circRNAs between mouse and human brain samples was examined using the UCSC liftOver tool as described in Veno et al. ${ }^{2}$. Twenty base pairs from each end of circRNAs were lifted from mm10 to hg19 or from hg19 to mm10, recombined and compared to circRNAs sequenced from the relevant species. This approach allows conversion of circRNA BSJ genomic coordinates between mouse and human genomes without requirements for internal splicing patterns of the circRNAs. CircRNAs are conserved when conversion between mouse and human genomes have exact matches to detected circRNAs in both datasets.

Detection of intron usage in circRNAs. In order to detect potential intron retention in circRNAs, we detect the percentage of intron mapping bases from BSJ spanning reads for each annotated intron within circRNA genomic regions. This data was collected for each read individually, showing how many bp each individual BSJ spanning read maps to Gencode annotated exons and introns. This allows us to calculate the intron coverage for each circRNA, defined as the mean number of intron mapping bp in the BSJ spanning reads that define the circRNA divided by the mean number of gene mapping bp in the BSJ spanning reads.

Introns within circRNA genomic regions that are covered by 20 or more BSJ reads, have read coverage across $90 \%$ or more of the intron length and no detection of previously unannotated (novel) exons are called as retained introns.

Novel exons. Exons present in circRNAs but not annotated in either Gencode or RefSeq were detected by scanning mapped Nanopore reads for flanking AG-GT splicing signatures in the reference genome. Read segments mapping in discrete blocks, with allowance of up to $10 \mathrm{bp}$ internal deletion, were checked for flanking base pairs. Segments that are flanked by AG on the 5' side and GT on the 3' side, consistent with RNA splicing, are marked as likely exons. If two or more reads corroborate the exon definition, it is considered a bona fide exon. No end processing of the Nanopore reads is done before detecting flanking sequences. Detected circRNA exons that do not match an annotated exon by at least $95 \%$ similarity on genome coordinates are considered novel exons.

Note that only circRNAs with two or more exons contribute to this analysis, since BSJ spanning reads are required to completely cover an exon in a single genomic mapping location. Single-exon circRNAs are combined from two genomic mapping locations of individual BSJ spanning reads, and do not count in this analysis. 
Novel exons that do not overlap any annotated exon are classified as "unique exons", whereas exons that partially overlap an already annotated exon are classified as "cryptic exons", since these exons presumably arise from cryptic splice sites not normally used in the linear mRNA host (Fig. 5a).

Novel exons are investigated for their ability to introduce stop codons. In order to do this, the codons for each of the three possible phases were prepared for all novel exons and the presence of stop codons in each of the three phases was detected. By use of a custom script Gencode gene annotations were processed to get the phase used by each annotated exon. The phase used by the immediate upstream exon dictates the phase used by a novel exon. If the phase in use by a novel exon contains a stop codon, insertion of this exon in a protein coding mRNA would disrupt transcript function, but insertion in a non-protein-coding circRNA such a stop codon would have no consequence.

Alternative exon usage. The usage of exons within the mapping range of each individual BSJ spanning read is detected and used to build alternative exon usage tables for each circRNA. For each circRNA, we quantify the number of BSJ reads mapping to genomic coordinates, including specific exons and how many of these BSJ reads have sequence mapping to the exon. Exon usage level is then defined as the number of BSJ reads using the exon divided by the number of BSJ reads mapping across the exon. Only exons used in 10 or more reads for circNick-LRS or 50 or more for circPanel-LRS are included in the analysis. Exons that are constitutively used in circRNAs have an exon usage level of 1 , whereas alternatively used exons have an exon usage level between 0 and 1 .

FLAIR analysis of ONT CDNA-PCR sequencing data. Data was basecalled using Guppy (v 3.4.5). Basecalled fastq files were analyzed using FLAIR v 1.4 (FullLength Alternative Isoform analysis of RNA $)^{30}$, performing genome alignment and end correction of the ONT cDNA-PCR reads. The corrected reads were collapsed to generate complete lists of isoforms detected.

Short RNA sequencing data analysis. Adapter sequences and low quality reads were trimmed away using Trim Galore (v 0.4.1). Short RNA sequencing data was quality controlled using FastQC (v 0.11.5). Detection of circRNAs was primarily done using CIRI2 (v 2.0.6) and secondarily by find_circ (v 1), which was used to corroborate circRNA detection by CIRI2. Positive detection of circRNAs by two different algorithms is know to reduce false circRNA detection.

Reporting summary. Further information on research design is available in the Nature Research Reporting Summary linked to this article.

\section{Data availability}

The data supporting the findings of this study are available from the corresponding authors upon reasonable request. All the sequencing data generated in this study have been deposited to the Gene Expression Omnibus (GEO) repository database with the accession number GSE127059. The following circRNA databases were used: circBase (http://www.circbase.org/), CIRCpedia (https://www.picb.ac.cn/rnomics/circpedia/), and CircAtlas (http://circatlas.biols.ac.cn/) ${ }^{33-35}$. Source data file is provided.

\section{Code availability}

Custom scripts used in this manuscript and a minimal test dataset can be found on github: https://github.com/omiics-dk/long_read_circRNA.

Received: 12 January 2021; Accepted: 16 July 2021;

Published online: 10 August 2021

\section{References}

1. Rybak-Wolf, A. et al. Circular RNAs in the mammalian brain are highly abundant, conserved, and dynamically expressed. Mol. Cell 58, 870-885 (2014).

2. Venø, M. T. et al. Spatio-temporal regulation of circular RNA expression during porcine embryonic brain development. Genome Biol. 16, 245 (2015).

3. Ashwal-Fluss, R. et al. CircRNA biogenesis competes with pre-mRNA splicing. Mol. Cell 56, 55-66 (2014).

4. Liu, C. X. et al. Structure and degradation of circular RNAs regulate PKR activation in innate immunity. Cell 177, 865-880.e21 (2019).

5. Kristensen, L., Hansen, T., Venø, M. \& Kjems, J. Circular RNAs in cancer: opportunities and challenges in the field. Oncogene 37361, 555-565 (2018).

6. Okholm, T. L. H. et al. Circular RNA expression is abundant and correlated to aggressiveness in early-stage bladder cancer. npj Genom. Med. 2, 36 (2017).

7. Vidal, A. F. et al. The comprehensive expression analysis of circular RNAs in gastric cancer and its association with field cancerization. Sci. Rep. 7, 14551 (2017).
8. Hansen, T. B. et al. Natural RNA circles function as efficient microRNA sponges. Nature 495, 384-388 (2013).

9. Kleaveland, B., Shi, C. Y., Stefano, J. \& Bartel, D. P. A network of noncoding regulatory RNAs acts in the mammalian brain. Cell 174, 350-362.e17 (2018).

10. Memczak, S. et al. Circular RNAs are a large class of animal RNAs with regulatory potency. Nature 495, 333-338 (2013).

11. Piwecka, M. et al. Loss of a mammalian circular RNA locus causes miRNA deregulation and affects brain function. Science 357, eaam8526-eaam8526 (2017).

12. Hansen, T. B., Venø, M. T., Damgaard, C. K. \& Kjems, J. Comparison of circular RNA prediction tools. Nucleic Acids Res. 44, gkv1458-gkv1458 (2015).

13. Li, S. et al. Microarray is an efficient tool for circRNA profiling. Brief. Bioinform. https://doi.org/10.1093/bib/bby006 (2018).

14. Zhang, S. et al. Microarray profile of circular RNAs identifies hsa_circ_0014130 as a new circular RNA biomarker in non-small cell lung cancer OPEN. Sci. Rep. 8, 2878 (2018).

15. Dahl, M. et al. Enzyme-free digital counting of endogenous circular RNA molecules in B-cell malignancies. Lab. Investig. https://doi.org/10.1038/ s41374-018-0108-6 (2018).

16. Gao, Y. et al. Comprehensive identification of internal structure and alternative splicing events in circular RNAs. Nat. Commun. 7, 12060 (2016).

17. Zheng, Y., Ji, P., Chen, S., Hou, L. \& Zhao, F. Reconstruction of full-length circular RNAs enables isoform-level quantification. Genome Med. 11, 2 (2019).

18. Check Hayden, E. Data from pocket-sized genome sequencer unveiled. Nature https://doi.org/10.1038/nature.2014.14724 (2014).

19. Rhoads, A. \& Au, K. F. PacBio sequencing and its applications. Genom. Proteom. Bioinform. 13, 278-289 (2015).

20. Byrne, A. et al. Nanopore long-read RNAseq reveals widespread transcriptional variation among the surface receptors of individual B cells. Nat. Commun. 8, 16027 (2017).

21. Chuang, T.-J. et al. Integrative transcriptome sequencing reveals extensive alternative trans-splicing and cis-backsplicing in human cells. Nucleic Acids Res. https://doi.org/10.1093/nar/gky032 (2018).

22. Jain, M. et al. Nanopore sequencing and assembly of a human genome with ultra-long reads. Nat. Biotechnol. 36, 338-345 (2018).

23. Oikonomopoulos, S., Wang, Y. C., Djambazian, H., Badescu, D. \& Ragoussis, J. Benchmarking of the Oxford Nanopore MinION sequencing for quantitative and qualitative assessment of cDNA populations. Sci. Rep. 6, 31602 (2016).

24. Workman, R. E. et al. Nanopore native RNA sequencing of a human poly(A) transcriptome. Nat. Methods 16, 1297-1305 (2019).

25. Hirsch, S. et al. Circular RNAs of the nucleophosmin (NPM1) gene in acute myeloid leukemia. Haematologica 102, 2039-2047 (2017).

26. Rahimi, K., Venø, M., Dupont, D. \& Kjems, J. Nanopore sequencing of full-length circRNAs in human and mouse brains reveals circRNA-specific exon usage and intron retention. bioRxiv https://doi.org/10.1101/567164 (2019).

27. Gonatopoulos-Pournatzis, T. \& Blencowe, B. J. Microexons: at the nexus of nervous system development, behaviour and autism spectrum disorder. Curr. Opin. Genet. Dev. 65, 22-33 (2020).

28. Gruner, H., Cortés-López, M., Cooper, D. A., Bauer, M. \& Miura, P. CircRNA accumulation in the aging mouse brain. Sci. Rep. 6, 38907 (2016).

29. Panda, A. C. et al. High-purity circular RNA isolation method (RPAD) reveals vast collection of intronic circRNAs. Nucleic Acids Res. 45, e116 (2017).

30. Tang, A. D. et al. Full-length transcript characterization of SF3B1 mutation in chronic lymphocytic leukemia reveals downregulation of retained introns. Nat. Commun. 11, 1438 (2020).

31. Gao, Y., Zhang, J. \& Zhao, F. Circular RNA identification based on multiple seed matching. Brief Bioinform. 19, 803-810 (2018).

32. Jeck, W. R. et al. Circular RNAs are abundant, conserved, and associated with ALU repeats. RNA 19, 141-157 (2013).

33. Glažar, P., Papavasileiou, P. \& Rajewsky, N. CircBase: a database for circular RNAs. RNA 20, 1666-1670 (2014).

34. Wu, W., Ji, P. \& Zhao, F. CircAtlas: an integrated resource of one million highly accurate circular RNAs from 1070 vertebrate transcriptomes. Genome Biol. 21, 101 (2020).

35. Dong, R., Ma, X. K., Li, G. W. \& Yang, L. CIRCpedia v2: an updated database for comprehensive circular RNA annotation and expression comparison. Genom. Proteom. Bioinform. 16, 226-233 (2018).

36. Hansen, T. B. et al. miRNA-dependent gene silencing involving Ago2mediated cleavage of a circular antisense RNA. EMBO J. 30, 4414-4422 (2011).

37. Barrett, S. P., Parker, K. R., Horn, C., Mata, M. \& Salzman, J. ciRS-7 exonic sequence is embedded in a long non-coding RNA locus. PLoS Genet. 13, e1007114 (2017).

38. Irimia, M. et al. A highly conserved program of neuronal microexons is misregulated in autistic brains. Cell 159, 1511-1523 (2014). 
39. Gonatopoulos-Pournatzis, T. et al. Autism-misregulated eIF4G microexons control synaptic translation and higher order cognitive functions. Mol. Cell 77, 1176-1192.e16 (2020).

40. $\mathrm{Li}, \mathrm{Z}$. et al. Exon-intron circular RNAs regulate transcription in the nucleus. Nat. Struct. Mol. Biol. 22, 256-264 (2015).

41. Mauger, O., Lemoine, F. \& Scheiffele, P. Targeted intron retention and excision for rapid gene regulation in response to neuronal activity. Neuron $\mathbf{9 2}$, 1266-1278 (2016).

42. Wong, J. J. L., Au, A. Y. M., Ritchie, W. \& Rasko, J. E. J. Intron retention in mRNA: no longer nonsense: Known and putative roles of intron retention in normal and disease biology. BioEssays 38, 41-49 (2016).

43. Belgrader, P., Cheng, J. \& Maquat, L. E. Evidence to implicate translation by ribosomes in the mechanism by which nonsense codons reduce the nuclear level of human triosephosphate isomerase mRNA. Proc. Natl Acad. Sci. USA 90, 482-486 (1993).

44. Lykke-Andersen, S. \& Jensen, T. H. Nonsense-mediated mRNA decay: an intricate machinery that shapes transcriptomes. Nat. Rev. Mol. Cell Biol. 16, 665-677 (2015).

45. Jaffrey, S. R. \& Wilkinson, M. F. Nonsense-mediated RNA decay in the brain: emerging modulator of neural development and disease. Nat. Rev. Neurosci. 19, 715-728 (2018).

46. Hansen, T. B. Signal and noise in circRNA translation. Methods https://doi. org/10.1016/j.ymeth.2021.02.007 (2021).

47. Ustianenko, D., Weyn-Vanhentenryck, S. M. \& Zhang, C. Microexons: discovery, regulation, and function. Wiley Interdiscip. Rev. RNA 8, e1418 (2017).

48. Curry-Hyde, A., Chen, B. J., Mills, J. D. \& Janitz, M. Microexons: novel regulators of the transcriptome. J. Hum. Transcr. 2, 1-6 (2018).

49. Ebbesen, K. K., Kjems, J. \& Hansen, T. B. Circular RNAs: Identification, biogenesis and function. Biochim. Biophys. Acta 1859, 163-168 (2016).

50. Kristensen, L. S. et al. The biogenesis, biology and characterization of circular RNAs. Nat. Rev. Genet. 20, 675-691 (2019).

51. Xin, R. et al. isoCirc catalogs full-length circular RNA isoforms in human transcriptomes. Nat. Commun. 12, 266 (2021).

52. Zhang, J. et al. Comprehensive profiling of circular RNAs with nanopore sequencing and CIRI-long. Nat. Biotechnol. https:/doi.org/10.1038/s41587021-00842-6 (2021).

53. Kent, W. J. BLAT - The BLAST-like alignment tool. Genome Res. 12, 656-664 (2002).

54. Wang, M. \& Kong, L. pblat: A multithread blat algorithm speeding up aligning sequences to genomes. BMC Bioinform. 20, 28 (2019).

\section{Acknowledgements}

We acknowledge the support of this project by Danish Council for Independent Research (4181-00446B), the Novo Nordisk Foundation (NNF18OC0032824), and the Villum
Foundation (00013393). We are grateful to Anne Færch Nielsen and Thomas Birkballe Hansen for editing and critical reading of the manuscript.

\section{Author contributions}

K.R. and J.K. provided the conceptual idea and designed the study. KR enriched, designed, and prepared compatible circRNA libraries for ONT sequencing. K.R. and D.M.D. optimized and developed ONT full-length circRNA sequencing. K.R. designed and optimized the circRNA full-length panel sequencing. M.T.V. performed bioinformatics analysis and wrote the scripts. K.R. and M.T.V. performed data analysis and drafted the manuscript. K.R., M.T.V., D.M.D., and J.K. edited and approved the manuscript and contributed to the project by significant insights.

\section{Competing interests}

The authors declare no competing interests.

\section{Additional information}

Supplementary information The online version contains supplementary material available at https://doi.org/10.1038/s41467-021-24975-z.

Correspondence and requests for materials should be addressed to K.R. or J.K.

Peer review information Nature Communications thanks the anonymous reviewers for their contributions to the peer review of this work.

Reprints and permission information is available at http://www.nature.com/reprints

Publisher's note Springer Nature remains neutral with regard to jurisdictional claims in published maps and institutional affiliations.

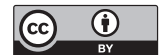

Open Access This article is licensed under a Creative Commons Attribution 4.0 International License, which permits use, sharing, adaptation, distribution and reproduction in any medium or format, as long as you give appropriate credit to the original author(s) and the source, provide a link to the Creative Commons license, and indicate if changes were made. The images or other third party material in this article are included in the article's Creative Commons license, unless indicated otherwise in a credit line to the material. If material is not included in the article's Creative Commons license and your intended use is not permitted by statutory regulation or exceeds the permitted use, you will need to obtain permission directly from the copyright holder. To view a copy of this license, visit http://creativecommons.org/ licenses/by/4.0/.

(C) The Author(s) 2021 